JOURNAL OF THE

AMERICAN MATHEMATICAL SOCIETY

Volume 21, Number 4, October 2008, Pages 1169-1209

S 0894-0347(08)00597-3

Article electronically published on February 12, 2008

\title{
DISK ENUMERATION ON THE QUINTIC 3-FOLD
}

\author{
R. PANDHARIPANDE, J. SOLOMON, AND J. WALCHER
}

\section{INTRODUCTION}

0.1. Complex curve enumeration. Let $Q \subset \mathbb{C P}^{4}$ be a nonsingular quintic hypersurface. The virtual count $n_{d}$ of rational algebraic curves of degree $d>0$ on $Q$ admits a computation via Gromov-Witten theory and mirror symmetry.

Let $N_{d}$ denote the genus 0 Gromov-Witten invariant of $Q$ in degree $d$. The count $n_{d}$ is defined by the Aspinwall-Morrison formula [1]

$$
\sum_{d>0} N_{d} e^{d T}=\sum_{d>0} \sum_{k>0} n_{d} k^{-3} e^{k d T} .
$$

The connection between $n_{d}$ and actual curve counting on $Q$ is discussed in [16].

The mirror symmetry prediction of Candelas, de la Ossa, Green, and Parkes 2 relates the genus 0 potential

$$
\mathcal{F}(T)=\frac{5}{6} T^{3}+\sum_{d>0} N_{d} e^{d T}
$$

to hypergeometric series. Let $I_{i}(t)$ be defined by

$$
\sum_{i=0}^{3} I_{i} H^{i}=\sum_{d=0}^{\infty} e^{(H+d) t} \frac{\Pi_{r=1}^{5 d}(5 H+r)}{\Pi_{r=1}^{d}(H+r)^{5}} \bmod H^{4} .
$$

The functions $I_{i}(t)$ are a basis of solutions of the Picard-Fuchs differential equation

$$
\left(\frac{d}{d t}\right)^{4} I-5 e^{t}\left(5 \frac{d}{d t}+1\right)\left(5 \frac{d}{d t}+2\right)\left(5 \frac{d}{d t}+3\right)\left(5 \frac{d}{d t}+4\right) I=0 .
$$

Let the variables $T$ and $t$ be related by $T(t)=I_{1} / I_{0}(t)$. The prediction,

$$
\mathcal{F}(T(t))=\frac{5}{2}\left(\frac{I_{1}}{I_{0}}(t) \frac{I_{2}}{I_{0}}(t)-\frac{I_{3}}{I_{0}}(t)\right),
$$

was later proven via localization on the space of genus 0 stable maps to $\mathbb{C P}^{4}[5$, 11 , 13.

Received by the editors May 29, 2007.

2000 Mathematics Subject Classification. Primary 53D45, 14N35; Secondary 14J32.

(C)2008 American Mathematical Society Reverts to public domain 28 years from publication 
0.2. Disk enumeration. Let $Q \subset \mathbb{C P}^{4}$ be a nonsingular quintic hypersurface defined over $\mathbb{R}$. Let $\omega$ be the symplectic form on $Q$ obtained from the Fubini-Study metric. Complex conjugation determines an anti-holomorphic involution on $Q$ with fixed locus equal to the set of real points $Q_{\mathbb{R}}$. The inclusion

$$
Q_{\mathbb{R}} \subset Q
$$

is Lagrangian with respect to $\omega$.

The enumeration of disks with boundaries in Lagrangian submanifolds plays a basic role in open string theory and has been studied mathematically in several contexts. The subject is not a direct extension of the theory of stable maps. New issues such as orientation play a crucial role. Here we provide a complete calculation of the disk invariants of $Q$ with boundary in the real Lagrangian $Q_{\mathbb{R}}$.

An early treatment of disk enumeration occurs in the construction of the Fukaya category [3]. Disk enumeration is required to define the differentials of the Floer complex. However, a symplectic invariant via disk enumeration is not defined in 3. Only the cohomology of the Floer complex is invariant.

Symplectic disk invariants have been defined with respect to the real Lagrangian associated to an anti-holomorphic involution in 19. A previous definition in the presence of a torus action preserving the real Lagrangian (not directly applicable to $Q$ ) can be found in [10, 14. Here we will follow the definitions of [19].

Let $N_{d}^{d i s k}$ for $d$ odd denote the degree $d$ disk invariant of $Q$ with boundary in $Q_{\mathbb{R}}$. For a discussion of even degree, see Sections 0.4 and 1.5. In fact, $N_{d}^{d i s k}$ depends on a choice of a Spin structure on $Q_{\mathbb{R}}$. However, changing the Spin structure only effects the sign of $N_{d}^{\text {disk }}$ uniformly for all $d$. Our conventions are fixed by choosing $N_{1}^{\text {disk }}$ to be positive. Let $\mathcal{F}^{\text {disk }}$ denote the disk potential,

$$
\mathcal{F}^{\text {disk }}(T)=\sum_{d \text { odd }} N_{d}^{d i s k} e^{d T / 2}
$$

Our main result is a calculation of $\mathcal{F}^{\text {disk }}$. Define

$$
J(t)=2 \sum_{d \text { odd }} e^{d t / 2} \frac{(5 d) ! !}{(d ! !)^{5}} .
$$

The function $J(t)$ is a solution of the Picard-Fuchs equation (21) with an added inhomogenous term,

$$
\left(\frac{d}{d t}\right)^{4} J-5 e^{t}\left(5 \frac{d}{d t}+1\right)\left(5 \frac{d}{d t}+2\right)\left(5 \frac{d}{d t}+3\right)\left(5 \frac{d}{d t}+4\right) J=\frac{15}{8} e^{t / 2} .
$$

Alternatively, $J(t)$ may be obtained by evaluation at $H=1 / 2$ of the (nontruncated) hypergeometric solution (11) of the homogeneous Picard-Fuchs equation,

$$
J(t)=30\left[\sum_{d=0}^{\infty} e^{(H+d) t} \frac{\Pi_{r=1}^{5 d}(5 H+r)}{\Pi_{r=1}^{d}(H+r)^{5}}\right]_{H=\frac{1}{2}} .
$$

Theorem 1. Via the mirror map $T(t)=I_{1} / I_{0}(t)$,

$$
\mathcal{F}^{\text {disk }}(T(t))=\frac{J(t)}{I_{0}(t)}
$$

The disk invariants $N_{d}^{d i s k}$ are typically fractional. Following the strategy of curve enumeration, virtual disk counts $n_{d}^{\text {disk }}$ are defined by the Ooguri-Vafa formula. 
Definition 2. We define the counts $n_{d}^{\text {disk }}$ by

$$
\sum_{d \text { odd }} N_{d}^{d i s k} e^{d T / 2}=\sum_{d \text { odd }} \sum_{k \text { odd }} n_{d / k}^{d i s k} k^{-2} e^{k d T / 2} .
$$

Definition 2 is justified by the multiple cover calculation of Proposition 19 in Section 6. The contribution of $k$-fold covers of a disk in the appropriate local Calabi-Yau geometry is $k^{-2}$. We conjecture the invariants $n_{d}^{\text {disk }}$ to be integers.

0.3. Real curve enumeration. Each holomorphic disk with boundary in $Q_{\mathbb{R}}$ can be reflected by the Schwartz principle to yield a real rational curve in $Q$. Conversely, real curves mapping to $Q$ of odd degree may be halved to yield two disks [19]. The virtual number of real rational curves of odd degree in $Q$ may be defined by

$$
n_{d}^{\text {real }}=\frac{1}{2} n_{d}^{\text {disk }}
$$

Again, $n_{d}^{\text {real }}$ vanishes for $d$ even. A table of values can be found at the end of the paper.

0.4. Mirror symmetry. Let $\mathcal{L}$ be a $U(1)$ bundle with flat connection $A_{0}$ over the Lagrangian submanifold $Q_{\mathbb{R}} \subset Q$. The triple

$$
\mathcal{O}=\left(Q_{\mathbb{R}}, \mathcal{L}, A_{0}\right)
$$

determines an object of the Fukaya category of $Q$. Homological mirror symmetry 12 predicts the existence of a corresponding object $\mathcal{O}^{\vee}$ of the derived category of coherent sheaves on the mirror quintic $Q^{\vee}$. The holomorphic Chern-Simons functional of $\mathcal{O}^{\vee}$ is predicted to be mirror to the standard Chern-Simons functional of $\mathcal{O}$ with corrections from disk instantons [25]. In the following, we briefly explain how the mirror correspondence between functionals leads to an enumerative correspondence.

Assume for simplicity that $\mathcal{O}^{\vee}$ is a holomorphic vector bundle. Denote the underlying complex vector bundle by $V$, and let $A_{\mathcal{O}} \vee$ be the connection on $V$ defining the holomorphic structure of $\mathcal{O}^{\vee}$. Let $t$ denote the complex moduli parameter of $Q^{\vee}$, and let $\Omega_{t}$ denote the holomorphic 3-form determined by $t$. The holomorphic Chern-Simons functional of $V$ depends on a second complex connection on $V$, which we denote $A$. We view $A$ as a connection 1-form relative to $A_{\mathcal{O}^{\vee}}$. Define the holomorphic Chern-Simons functional by

$$
L^{\vee}(A, t)=\int_{Q^{\vee}} \operatorname{Tr}\left(A \wedge \bar{\partial}_{A_{\mathcal{O} \vee}} A+\frac{2}{3} A \wedge A \wedge A\right) \wedge \Omega_{t}
$$

Critical points of $L^{\vee}$ are holomorphic connections - complex connections with vanishing $(0,2)$ component of their curvature. $L^{\vee}$ is constant on connected components of orbits of the complex gauge group of $V$.

For the Chern-Simons functional with instanton corrections, we will require the following terminology for holonomy. If

$$
E \rightarrow B
$$

is a bundle with connection $\theta$, and $P$ is a parametrized path in $B$, we denote the holonomy of $\theta$ around $P$ by $\operatorname{Hol}(P, \theta)$. If $B \subset Q$ and

$$
f:\left(D^{2}, \partial D^{2}\right) \rightarrow(Q, B)
$$


we write

$$
P_{f}=\left\{\left.f\right|_{\partial D^{2}}: \partial D^{2} \rightarrow B\right\} .
$$

Moreover, if $f$ is holomorphic, we define $\eta_{f}$ to be the sign of $f$ coming from the determinant line of the Cauchy-Riemann operator.

In defining the Chern-Simons functional with instanton corrections, we will not try to be entirely precise, but rather give an intuitive picture. Let $M \subset Q$ be a totally real submanifold isotopic to $Q_{\mathbb{R}}$, and let $C^{4}$ be the cobordism traced out by the isotopy. In particular, $\partial C^{4}=M-Q_{\mathbb{R}}$. Let

$$
\mathcal{L}_{M} \rightarrow M
$$

be a $U(1)$ bundle, and let $A_{M}$ be a connection on $\mathcal{L}_{M}$. Since $M$ is naturally identified with $Q_{\mathbb{R}}$, even though $\mathcal{L}_{M}$ may be a different topological bundle than $\mathcal{L}$, we may consider $A_{0}$ as a perhaps singular connection on $\mathcal{L}_{M}$. Hence, we may think of $A_{M}$ as a connection 1 -form relative to $A_{0}$. Moreover, let us choose an embedded disk

$$
\left(C^{2}, \partial C^{2}\right) \subset(Q, M),
$$

such that $\partial C^{2}$ represents the Poincaré duals of the difference of first Chern classes $c_{1}(\mathcal{L})-c_{1}\left(\mathcal{L}_{M}\right)$. Choose an extension $\tilde{A}_{M}$ of the connection 1 -form $A_{M}$ to the disk $C^{2}$. Let $T$ denote the complexified Kähler moduli parameter of $Q$ and let $\omega_{T}$ denote the associated complexified Kähler form. Define the Chern-Simons function with instanton corrections by

$$
\begin{aligned}
L\left(M, A_{M}, T\right)= & \int_{M} A_{M} \wedge\left(d A_{M}-\omega_{T}\right)+\int_{C^{2}}\left(\omega_{T}+d \tilde{A}_{M}\right)+\int_{C^{4}} \omega_{T}^{2} \\
& +\sum_{f:\left(D^{2}, \partial D^{2}\right) \rightarrow(Q, M)} \eta_{f} \operatorname{Hol}\left(P_{f}, A_{M}\right) \exp \left(-\int_{D^{2}} f^{*} \omega_{T}\right) \\
& -\sum_{f:\left(D^{2}, \partial D^{2}\right) \rightarrow\left(Q, Q_{\mathbb{R}}\right)} \eta_{f} \operatorname{Hol}\left(P_{f}, A_{0}\right) \exp \left(-\int_{D^{2}} f^{*} \omega_{T}\right) \\
& +\sum_{\substack{f: S^{2} \rightarrow Q \\
\bar{\partial} f=0, z \in f^{-1}\left(C^{4}\right)}} \eta_{f} \exp \left(-\int_{S^{2}} f^{*} \omega_{T}\right) .
\end{aligned}
$$

A Lagrangian submanifold $M$ with vanishing obstruction chains in the sense of 3 ] and a flat connection $A_{M}$ together constitute a critical point of $L$. $L$ is constant on orbits of the Hamiltonian symplectomorphism group. The corrections from closed instantons intersecting $C^{4}$ are necessary to compensate for codimension one bubbling where all the energy of a disk instanton is transferred to a sphere bubble [18.

$L$ and $L^{\vee}$ are Lagrangians defining a pair of dual quantum field theories [25]. Their critical values are physically significant and should be topological invariants. Via the mirror transformation, which expresses $T$ as a function of $t$, the value of $L$ at a critical point should be calculable from the value of $L^{\vee}$ at a mirror critical point. However, we must find nontrivial choices of critical points $A$ and $\left(M, \mathcal{L}_{M}, A_{M}\right)$ corresponding under mirror symmetry for all values of $T=T(t)$. 
In our case, such $\left(M, \mathcal{L}_{M}, A_{M}\right)$ can be found using the geometry of the antiholomorphic involution. We choose

$$
M=Q_{\mathbb{R}} .
$$

Since $H_{1}\left(Q_{\mathbb{R}}\right)=\mathbb{Z} / 2$, we can choose $\left(\mathcal{L}_{M}, A_{M}\right)$ to be the flat $U(1)$ bundle with monodromy opposite to $\left(\mathcal{L}, A_{0}\right)$. Since $Q_{\mathbb{R}}$ is the fixed point set of an anti-holomorphic involution, $Q_{\mathbb{R}}$ is a critical point of $L$ with either flat bundle. The mirror to such a choice of $\left(\mathcal{L}_{M}, A_{M}\right)$ should be a unique up to gauge transformation holomorphic connection $A$ on $V$ not gauge equivalent to $A_{\mathcal{O}^{v}}$. In [21, a heuristic argument is given to show $L^{\vee}(A, t)$ is in fact given by $J(t)$. Even after specifying which holomorphic structure $A$ induces, $L^{\vee}(A, t)$ is only defined up to a period of $\Omega_{t}$. Indeed, changing the choice of $A$ by a complex gauge transformation not isotopic to the identity changes $L^{\vee}(A, t)$ by a period of $\Omega_{t}$. Therefore, $L^{\vee}(A, t)$ should satisfy an extension of the Picard-Fuchs equation for $Q^{\vee}$. Similarly, changing the choice of $C^{4}$ in the definition of $L$ changes $L$ by a multiple of the first derivative of $\mathcal{F}(T)$, which corresponds to a period under the mirror transform. Changing the choice of $C^{2}$ changes $L$ by a multiple of $T$, and changing $A_{M}$ by a large gauge transformation changes $L$ by a multiple of 1 , both of which correspond to periods under the mirror transform.

From the preceding discussion, we see both $L$ and $L^{\vee}$ are essentially relative functionals, depending either on a pair of connections, $A, A_{\mathcal{O}}$, or a pair of totally real submanifolds with $U(1)$ bundle, $\left(M, \mathcal{L}_{M}\right)$ and $\left(Q_{\mathbb{R}}, \mathcal{L}\right)$. Therefore only disks of odd degree, for which the difference in monodromy of $A_{M}$ and $A_{0}$ cancels the negative sign in definition (3), contribute to the physically significant critical value of $L^{\vee}$. The contributions of even degree disks cancel due to this sign. A priori, some other physical value may depend on the even degree disks. However, on mathematical grounds, even degree disks appear not to lead to interesting invariants; see Section 1.5 .

0.5. Past and future work. The first number $n_{1}^{\text {disk }}$ was calculated in [19. Theorem 1 was predicted in [21] via low degree graphs sums and string heuristics. Our technique of proof uses the fully equivariant mirror correspondence of Givental [5]. A previous application can be found in [7] where disk enumeration for (noncompact) local geometries was considered. The Ooguri-Vafa [15] multiple cover formula of Definition 2 is by now established in many settings; see [10, 14].

We have chosen the quintic 3-fold as our first case of study, but the methods of the paper are much more generally applicable. It will be interesting to see which aspects of the solution persist.

\section{Disk INVARIANTS}

1.1. Overview. We recall the definition of the disk invariant $N_{d}^{\text {disk }}$ of the quintic from [19. Our conventions for conjugation, real structures, and stable disk maps are discussed in Section 1.3. The Euler class approach to $N_{d}^{\text {disk }}$ is presented in Section 1.4

1.2. Definitions. Fix a symplectic manifold $(X, \omega)$ of real dimension less than or equal to 6 with an anti-symplectic involution $\phi$,

$$
\phi^{*} \omega=-\omega .
$$


The fixed points $L=F i x(\phi)$ define a Lagrangian submanifold of $X$. A Pin structure and, if $L$ is orientable, an orientation on $L$ induce a natural relative orientation on the moduli space $\bar{M}_{D}(X / L, \beta)$ of stable disk maps to $(X, L)$ of degree $\beta$. Since $\bar{M}_{D}(X / L, \beta)$ is an orbifold with corners, the definition of cohomology classes on the moduli space yielding an analog of Gromov-Witten theory is not immediately clear. However, using $\phi$, certain corners of $\bar{M}_{D}(X / L, \beta)$ may be eliminated.

More precisely, the boundary of $\bar{M}_{D}(X / L, \beta)$ consists generically of stable disk maps with two disk components. Replacing one of the two components by the image under $\phi$ yields another two component map. We define an equivalence relation $\sim$ on the boundary of $\bar{M}_{D}(X / L, \beta)$ based on this correspondence. For certain components of the boundary, the relation $\sim$ preserves orientation. After quotienting by $\sim$ on these components, we obtain a new moduli space $\widetilde{M}_{D}(X / L, \beta)$ with fewer corners which is still relatively orientable. On $\widetilde{M}_{D}(X / L, \beta)$, many interesting cohomology classes can be defined. Consequently, a set of invariants are obtained of the triple $(X, \omega, \phi)$ reminiscent of standard Gromov-Witten invariants in many respects.

In good situations, the invariants obtained from $\widetilde{M}_{D}(X / L, d)$ are actually enumerative. For example, Welschinger's signed counts of real curves [22, 23] arise as specializations of the theory [19].

\subsection{Conventions.}

1.3.1. Coordinates. Let $z_{0}, \ldots, z_{4}$ be homogeneous coordinates on $\mathbb{C P}^{4}$. The standard complex conjugation $c_{\mathrm{Id}}$ on $\mathbb{C P}^{4}$ is

$$
\left[z_{0}, z_{1}, z_{2}, z_{3}, z_{4}\right] \stackrel{c_{\text {Id }}}{\longmapsto}\left[\bar{z}_{0}, \bar{z}_{1}, \bar{z}_{2}, \bar{z}_{3}, \bar{z}_{4}\right] .
$$

Each $g \in \mathbb{P} \mathbb{G L}_{5}$ yields an anti-holomorphic involution

$$
c_{g}=g^{-1} \circ c_{\mathrm{Id}} \circ g: \mathbb{C P}^{4} \rightarrow \mathbb{C P}^{4}
$$

equivalent to $c_{\mathrm{Id}}$. In particular, the anti-holomorphic involution $c$,

$$
\left[z_{0}, z_{1}, z_{2}, z_{3}, z_{4}\right] \stackrel{c}{\mapsto}\left[\bar{z}_{0}, \bar{z}_{2}, \bar{z}_{1}, \bar{z}_{4}, \bar{z}_{3}\right]
$$

is associated to the matrix

$$
\left(\begin{array}{ccccc}
1 & 0 & 0 & 0 & 0 \\
0 & 1 & 1 & 0 & 0 \\
0 & i & -i & 0 & 0 \\
0 & 0 & 0 & 1 & 1 \\
0 & 0 & 0 & i & -i
\end{array}\right) .
$$

Let $\mathbb{C P}_{\mathbb{R}}^{4} \subset \mathbb{C P}^{4}$ denote the fixed points of $c$. The involution $c$ will be most convenient for our calculation of disk invariants.

1.3.2. Real geometry. A homogeneous polynomial $F\left(z_{0}, z_{1}, z_{2}, z_{3}, z_{4}\right)$ on $\mathbb{C P}^{4}$ is defined over $\mathbb{R}$ if

$$
\overline{F(z)}=F(c(z)) .
$$

For example,

$$
z_{1}+z_{2} \text { and } i z_{1}-i z_{2}
$$

are both real linear polynomials. 
A subvariety of $V \subset \mathbb{C P}^{4}$ is defined over $\mathbb{R}$ if the ideal $I(V)$ is generated by real homogeneous functions. The lines

$$
\begin{aligned}
& L=\left\{\left[0, z_{1}, z_{2}, 0,0\right] \mid z_{1}, z_{2} \in \mathbb{C}\right\}, \\
& L^{\prime}=\left\{\left[0,0,0, z_{3}, z_{4}\right] \mid z_{3}, z_{4} \in \mathbb{C}\right\}
\end{aligned}
$$

are both defined over $\mathbb{R}$.

The involution $c$ lifts canonically to the line bundles

$$
\mathcal{O}_{\mathbb{C P}^{4}}(k) \rightarrow \mathbb{C P}^{4} .
$$

The linear polynomials (5) are elements of

$$
H^{0}\left(\mathbb{C P}^{4}, \mathcal{O}_{\mathbb{C P}^{4}}(1)\right)_{\mathbb{R}} \subset H^{0}\left(\mathbb{C P}^{4}, \mathcal{O}_{\mathbb{C P}^{4}}(1)\right)_{\mathbb{C}}
$$

the space of real sections.

1.3.3. Maps. Let $u, v$ be homogeneous coordinates on $\mathbb{C P}^{1}$. Let

$$
c: \mathbb{C P}^{1} \rightarrow \mathbb{C P}^{1}
$$

be the anti-holomorphic involution defined by

$$
[u, v] \stackrel{c}{\mapsto}[\bar{v}, \bar{u}] .
$$

The $c$-fixed points, $\mathbb{C P}_{\mathbb{R}}^{1} \subset \mathbb{C P}^{1}$, form a circle.

A holomorphic disk map

$$
f:(D, \partial D) \rightarrow\left(\mathbb{C P}^{4}, \mathbb{C P}_{\mathbb{R}}^{4}\right)
$$

can be reflected by the Schwartz principle to yield an algebraic map

$$
\tilde{f}: \mathbb{C P}^{1} \rightarrow \mathbb{C P}^{4} \text {. }
$$

By definition, the degree $d$ of the disk map equals the degree of $\tilde{f}$.

The map $\tilde{f}$ satisfies the following real condition:

$$
\tilde{f} \circ c=c \circ \tilde{f} .
$$

Conversely, every algebraic map

$$
\tilde{f}: \mathbb{C P}^{1} \rightarrow \mathbb{C P}^{4}
$$

satisfying (6) yields two disk maps with boundary $\partial D$ equal to $\mathbb{C P}_{\mathbb{R}}^{1}$. The image of $\tilde{f}$ is a real subcurve of $\mathbb{C P}^{4}$.

Similarly, a stable holomorphic disk map $f$ reflects to a stable genus 0 map $\tilde{f}$ satisfying the real condition (6) with respect to the natural extension of $c$ to degenerations of $\mathbb{C P}^{1}$. In fact, stability for $f$ can be defined by stability for $\tilde{f}$. We will also use the notation

$$
f:(D, \partial D) \rightarrow\left(\mathbb{C P}^{4}, \mathbb{C P}_{\mathbb{R}}^{4}\right)
$$

for the stable case where $D$ and $\partial D$ are possibly reducible. However, $\partial D$ is always connected.

In the odd degree case, every stable genus 0 map to $\mathbb{C P}^{4}$ defined over $\mathbb{R}$ is obtained by reflection. 
1.3.4. Moduli. Let $M_{D}\left(\mathbb{C P}^{4} / \mathbb{C P}_{\mathbb{R}}^{4}, d\right)$ denote the moduli space of unpointed disk maps of odd degree $d$. Reflection yields an étale double cover of smooth orbifolds

$$
\epsilon: M_{D}\left(\mathbb{C P}^{4} / \mathbb{C P}_{\mathbb{R}}^{4}, d\right) \rightarrow M_{\mathbb{R}}\left(\mathbb{C P}^{4}, d\right)
$$

where $M_{\mathbb{R}}\left(\mathbb{C P}^{4}, d\right)$ denotes the moduli space of unpointed genus 0 algebraic maps defined over $\mathbb{R}$. The real dimension of $M_{\mathbb{R}}\left(\mathbb{C P}^{4}, d\right)$ is $5 d+1$. In fact, $\epsilon$ is an orientation double cover [19].

Now let $\bar{M}_{D}\left(\mathbb{C P}^{4} / \mathbb{C P}_{\mathbb{R}}^{4}, d\right)$ denote the compactification of the moduli space $M_{D}\left(\mathbb{C P}^{4} / \mathbb{C P}_{\mathbb{R}}^{4}, d\right)$ by stable disk maps, and let $\bar{M}_{\mathbb{R}}\left(\mathbb{C P}^{4}, d\right)$ denote the space of unpointed genus 0 algebraic stable maps defined over $\mathbb{R}$. The moduli space $\bar{M}_{D}\left(\mathbb{C P}^{4} / \mathbb{C P}_{\mathbb{R}}^{4}, d\right)$ is a smooth orbifold with corners. In fact, $\epsilon$ extends to a finite smooth map

$$
\bar{\epsilon}: \bar{M}_{D}\left(\mathbb{C P}^{4} / \mathbb{C P}_{\mathbb{R}}^{4}, d\right) \rightarrow \bar{M}_{\mathbb{R}}\left(\mathbb{C P}^{4}, d\right),
$$

mapping the corners of $\bar{M}_{D}\left(\mathbb{C P}^{4} / \mathbb{C P}_{\mathbb{R}}^{4}, d\right)$ to the boundary divisor of $\bar{M}_{\mathbb{R}}\left(\mathbb{C P}^{4}, d\right)$. The cardinality of the fiber over a real stable map with $n_{o}$ components fixed by $c$ is $2^{n_{o}}$.

In Section 1.1, the construction of the closed orbifold

$$
\widetilde{M}_{D}\left(\mathbb{C P}^{4} / \mathbb{C P}_{\mathbb{R}}^{4}, d\right)=\bar{M}_{D}\left(\mathbb{C P}^{4} / \mathbb{C P}_{\mathbb{R}}^{4}, d\right) / \sim
$$

was outlined. A detailed argument is given in Section 5 in the proof of Proposition 11. The equivalence relation $\sim$ identifies the corners of $\bar{M}_{D}\left(\mathbb{C P}^{4} / \mathbb{C P}_{\mathbb{R}}^{4}, d\right)$ in such a way that the map $\bar{\epsilon}$ descends to an étale double cover

$$
\tilde{\epsilon}: \widetilde{M}_{D}\left(\mathbb{C P}^{4} / \mathbb{C P}_{\mathbb{R}}^{4}, d\right) \rightarrow \bar{M}_{\mathbb{R}}\left(\mathbb{C P}^{4}, d\right) .
$$

There is a natural inclusion

$$
\bar{M}_{\mathbb{R}}\left(\mathbb{C P}^{4}, d\right) \subset \bar{M}_{\mathbb{C}}\left(\mathbb{C P}^{4}, d\right)
$$

in the space of unpointed stable genus 0 algebraic maps defined over $\mathbb{C}$. The real dimension of $\bar{M}_{\mathbb{R}}\left(\mathbb{C P}^{4}, d\right)$ is $5 d+1$.

1.4. Euler class formula. Let $Q \subset \mathbb{C P}^{4}$ be a nonsingular quintic hypersurface defined over $\mathbb{R}$ with symplectic form obtained from the Fubini-Study metric. An anti-symplectic involution

$$
\phi: Q \rightarrow Q
$$

is defined by complex conjugation. The Lagrangian $F i x(\phi)$ is the real locus $Q_{\mathbb{R}}$.

We consider maps from the holomorphic disk $D$ to $Q$ of odd degree with boundary lying in $Q_{\mathbb{R}}$,

$$
\bar{M}_{D}\left(Q / Q_{\mathbb{R}}, d\right) \subset \bar{M}_{D}\left(\mathbb{C P}^{4} / \mathbb{C P}_{\mathbb{R}}^{4}, d\right)
$$

Since the expected dimension of the moduli space of maps to $Q / Q_{\mathbb{R}}$ is 0 , the relevant Gromov-Witten invariant $N_{d}^{\text {disk }}$ is simply the virtual cardinality.

To calculate $N_{d}^{\text {disk }}$, following [19], we first reformulate $N_{d}^{\text {disk }}$ as the integral of an Euler class of an obstruction bundle over the moduli space $\widetilde{M}_{D}\left(\mathbb{C P}^{4} / \mathbb{C P}_{\mathbb{R}}^{4}, d\right)$. Such integrals may be studied via fixed point localization. A similar approach was used by Kontsevich [11 in the closed case.

Let $\hat{F}_{d}$ be the real vector bundle over $\bar{M}_{D}\left(\mathbb{C P}^{4} / \mathbb{C P}_{\mathbb{R}}^{4}, d\right)$ with fiber

$$
\left.\hat{F}_{d}\right|_{\left[f:(D, \partial D) \rightarrow\left(\mathbb{C P}^{4}, \mathbb{C P}_{\mathbb{R}}^{4}\right)\right]}=H^{0}\left(C, \tilde{f}^{*} \mathcal{O}_{\mathbb{C P}^{4}}(5)\right)_{\mathbb{R}}
$$

where

$$
\left[\tilde{f}: C \rightarrow \mathbb{C P}^{4}\right] \in \bar{M}_{\mathbb{R}}\left(\mathbb{C P}^{4}, d\right)
$$


is the stable rational map obtained from the stable disk map via reflection, and $H^{0}\left(C, \tilde{f}^{*} \mathcal{O}_{\mathbb{C P}^{4}}(5)\right)_{\mathbb{R}}$ denotes real sections.

The vector bundle $\hat{F}_{d}$ is of real rank $5 d+1$ and is oriented on $\bar{M}_{D}\left(\mathbb{C P}^{4} / \mathbb{C P}_{\mathbb{R}}^{4}, d\right)$ by Lemma 8.7 of $\left[19\right.$. The integral of the Euler class $e\left(\hat{F}_{d}\right)$ over $\bar{M}_{D}\left(\mathbb{C P}^{4} / \mathbb{C P}_{\mathbb{R}}^{4}, d\right)$ is not well defined because the space $\bar{M}_{D}\left(\mathbb{C P}^{4} / \mathbb{C P}_{\mathbb{R}}^{4}, d\right)$ has nonempty boundary and $\hat{F}_{d}$ is not trivial near the boundary. However, $\hat{F}_{d}$ naturally descends to a vector bundle

$$
F_{d} \rightarrow \widetilde{M}_{D}\left(\mathbb{C P}^{4} / \mathbb{C P}_{\mathbb{R}}^{4}, d\right)
$$

Neither $F_{d}$ nor $\widetilde{M}_{D}\left(\mathbb{C P}^{4} / \mathbb{C P}_{\mathbb{R}}^{4}, d\right)$ are orientable. Let $\mathcal{L}$ be the local system defined by the determinant of the tangent bundle of the moduli space $\widetilde{M}_{D}\left(\mathbb{C P}^{4} / \mathbb{C P}_{\mathbb{R}}^{4}, d\right)$. In Lemma 13 of Section 5 , we prove

$$
\operatorname{det} F_{d} \simeq \mathcal{L}
$$

as topological bundles. A Spin structure on $Q_{\mathbb{R}}$ determines the choice of the isomorphism uniquely up to scaling by a positive constant. Hence, the Euler class

$$
e\left(F_{d}\right) \in H^{5 d+1}\left(\widetilde{M}_{D}\left(\mathbb{C P}^{4} / \mathbb{C P}_{\mathbb{R}}^{4}, d\right), \mathcal{L}\right)
$$

is well-defined. Since $\widetilde{M}_{D}\left(\mathbb{C P}^{4} / \mathbb{C P}_{\mathbb{R}}^{4}, d\right)$ is a closed orbifold, the integral

$$
\int_{\widetilde{M}_{D}\left(\mathbb{C P}^{4} / \mathbb{C P}_{\mathbb{R}}^{4}, d\right)} e\left(F_{d}\right)
$$

is well-defined. In Section [5, we obtain the following result.

Theorem 3. For $d$ odd,

$$
N_{d}^{\text {disk }}=\int_{\widetilde{M}_{D}\left(\mathbb{C P}^{4} / \mathbb{C P}_{\mathbb{R}}^{4}, d\right)} e\left(F_{d}\right) .
$$

We prove Theorem 3 using the symplectic virtual moduli cycle. The same technique can be used to prove the analogous well-known result for the closed invariants.

1.5. $N_{d}^{\text {disk }}$ Invariant in even degree. A stable disk map of even degree may still be reflected to obtain an even degree real genus 0 stable map. However, not all stable genus 0 maps of even degree defined over $\mathbb{R}$ are so obtained. Stable maps defined over $\mathbb{R}$ with domains having no real points cannot be halved.

The even disk invariant $N_{d}^{\text {disk }}$ is not well-defined without the addition of the contributions of real curves without real points. If such contributions were incorporated, $N_{d}^{\text {disk }}$ would be expressible as the Euler class of an odd-dimensional real bundle and hence would presumably vanish. Hence, the definition $N_{d}^{\text {disk }}=0$ for $d$ even.

1.6. Dependence on $Q$. Our formula for $N_{d}^{\text {disk }}$ is independent of the quintic $Q \subset$ $\mathbb{C P}^{4}$ defined over $\mathbb{R}$. Since the calculation is done on $\mathbb{C P}^{4}$, some information is possibly lost. More precisely, let

$$
\epsilon: H_{1}\left(Q_{\mathbb{R}}, \mathbb{Z} / 2 \mathbb{Z}\right) \rightarrow H_{1}\left(\mathbb{C P}_{\mathbb{R}}^{4}, \mathbb{Z} / 2 \mathbb{Z}\right) \cong \mathbb{Z} / 2 \mathbb{Z} .
$$

The invariant $N_{d}^{\text {disk }}$ is an integral over all stable disk maps

$$
f:(D, \partial D) \rightarrow\left(Q, Q_{\mathbb{R}}\right)
$$

of degree $d$ with boundary $\partial D$ determining a class in

$$
\epsilon^{-1}(\overline{1}) \subset H_{1}\left(Q_{\mathbb{R}}, \mathbb{Z} / 2 \mathbb{Z}\right) .
$$


If $\epsilon$ is an isomorphism, as is the case, for example, for the Fermat quintic

$$
Q=\left(z_{0}^{5}+z_{1}^{5}+z_{2}^{5}+z_{3}^{5}+z_{4}^{5}\right)
$$

then there is no loss of information. If, however, $\epsilon$ has a kernel, more refined disk invariants of $\left(Q, Q_{\mathbb{R}}\right)$ may sometimes be defined for

$$
\gamma \in \epsilon^{-1}(\overline{1}) \subset H_{1}\left(Q_{\mathbb{R}}, \mathbb{Z} / 2 \mathbb{Z}\right) .
$$

In the latter case,

$$
N_{d}^{d i s k}=\sum_{\gamma \in \epsilon^{-1}(\overline{1})} N_{d, \gamma}^{d i s k} .
$$

\section{TORus ACTIONS}

2.1. Tori. Let $\mathbf{T}$ denote the complex numbers of unit modulus,

$$
\mathbf{T}=\{\xi \in \mathbb{C}|| \xi \mid=1\} .
$$

The torus $\mathbf{T}^{5}$ acts diagonally on $\mathbb{C}^{5}$. A $\mathbf{T}^{5}$-action on $\mathbb{C P}^{4}$ is obtained by projectivization, and canonical lifts to the line bundles

$$
\mathcal{O}_{\mathbb{C P}^{4}}(k) \rightarrow \mathbb{C P}^{4}
$$

are obtained. There is a canonically induced translation action of $\mathbf{T}^{5}$ on $\bar{M}_{\mathbb{C}}\left(\mathbb{C P}^{4}, d\right)$.

Let $\zeta_{i} \in \mathbb{C P}^{4}$ denote the $\mathbf{T}^{5}$-fixed points,

$$
\zeta_{0}=[1,0,0,0,0], \zeta_{1}=[0,1,0,0,0], \ldots, \zeta_{5}=[0,0,0,0,1] .
$$

The involution $c$ fixes $\zeta_{0}$ and permutes the others:

$$
\zeta_{1} \stackrel{c}{\leftrightarrow} \zeta_{2}, \quad \zeta_{3} \stackrel{c}{\leftrightarrow} \zeta_{4}
$$

Hence, $\zeta_{0}$ is the unique real $\mathbf{T}^{5}$-fixed point.

Consider the rank 2 subtorus $\mathbf{T}^{2} \subset \mathbf{T}$ acting by

$$
\left(\xi_{1}, \xi_{2}\right) \cdot\left[z_{0}, z_{1}, z_{2}, z_{3}, z_{4}\right]=\left[z_{0}, \xi_{1} z_{1}, \bar{\xi}_{1} z_{2}, \xi_{2} z_{3}, \bar{\xi}_{2} z_{4}\right] .
$$

Since $\mathbf{T}^{2}$ preserves $\mathbb{C P}_{\mathbb{R}}^{4}$, translation defines a $\mathbf{T}^{2}$-action on the moduli spaces $\bar{M}_{D}\left(\mathbb{C P}^{4} / \mathbb{C P}_{\mathbb{R}}^{4}, d\right)$ and $\widetilde{M}_{D}\left(\mathbb{C P}^{4} / \mathbb{C P}_{\mathbb{R}}^{4}, d\right)$.

The algebraic torus $\left(\mathbb{C}^{*}\right)^{2}$ acts on $\mathbb{C P}^{4}$ by complexifying the action of $\mathbf{T}^{2}$,

$$
\left(\xi_{1}, \xi_{2}\right) \cdot\left[z_{0}, z_{1}, z_{2}, z_{3}, z_{4}\right]=\left[z_{0}, \xi_{1} z_{1}, \xi_{1}^{-1} z_{2}, \xi_{2} z_{3}, \xi_{2}^{-1} z_{4}\right] .
$$

Of course, $\left(\mathbb{C}^{*}\right)^{2}$ acts on $\bar{M}_{\mathbb{C}}\left(\mathbb{C P}^{4}, d\right)$ by translation.

2.2. Equivariant weights. We follow the equivariant weight conventions of [5, 16] for the torus $\mathbf{T}^{5}$.

Let $\lambda_{i}$ be the $\mathbf{T}^{5}$-equivariant cohomology class determined by the Chern class of the restriction of $\mathcal{O}_{\mathbb{C P}^{4}}(1)$ to $\zeta_{i}$ :

$$
\lambda_{i}=c_{1}\left(\mathcal{O}_{\mathbb{C P}^{4}}(1)_{\zeta_{i}}\right) \in H_{\mathbf{T}^{5}}^{*}(\mathrm{pt}) .
$$

The classes $\lambda_{i}$ generate

$$
H_{\mathbf{T}^{5}}^{*}(\mathrm{pt})=\mathbb{Q}\left[\lambda_{0}, \ldots, \lambda_{4}\right]
$$

The tangent weights of $\mathbb{C P}^{4}$ at the point $\zeta_{i}$ are $\left\{\lambda_{i}-\lambda_{j}\right\}_{j \neq i}$.

Let $\lambda, \lambda^{\prime}$ be the generators of $H_{\mathbf{T}^{2}}^{*}$ (pt) defined by the pull-back

$$
\rho^{*}: H_{\mathbf{T}^{5}}^{*}(\mathrm{pt}) \rightarrow H_{\mathbf{T}^{2}}^{*}(\mathrm{pt})
$$

and the equations

$$
\rho^{*}\left(\lambda_{1}\right)=-\rho^{*}\left(\lambda_{2}\right)=\lambda, \rho^{*}\left(\lambda_{3}\right)=-\rho^{*}\left(\lambda_{4}\right)=\lambda^{\prime} .
$$


The pull-back $\rho^{*}\left(\lambda_{0}\right)$ vanishes. For notational convenience, we will often omit the pull-back $\rho^{*}$ and write

$$
\lambda_{0}=0, \lambda_{1}=-\lambda_{2}=\lambda, \lambda_{3}=-\lambda_{4}=\lambda^{\prime} .
$$

2.3. Localization. The genus 0 Gromov-Witten invariants $N_{d}$ have been calculated in [5, 11, 13] via localization on $\bar{M}_{\mathbb{C}}\left(\mathbb{C P}^{4}, d\right)$ with respect to the $\mathbf{T}^{5}$-action. We will calculate $N_{d}^{d i s k}$ via localization on $\widetilde{M}_{D}\left(\mathbb{C P}^{4} / \mathbb{C P}_{\mathbb{R}}^{4}, d\right)$ with respect to the $\mathbf{T}^{2}$-action.

\section{Localization CALCUlation of $\mathcal{F}^{\text {disk }}$}

3.1. Overview. Let $d$ be odd. The $\mathbf{T}^{2}$-action on the moduli space $\widetilde{M}_{D}\left(\mathbb{C P} / \mathbb{C P}_{\mathbb{R}}^{4}, d\right)$ lifts canonically to the vector bundle $F_{d}$. We calculate the integral

$$
N_{d}^{d i s k}=\int_{\widetilde{M}_{D}\left(\mathbb{C P}^{4} / \mathbb{C P}^{4}, d\right)} e\left(F_{d}\right)
$$

by localization with respect to the $\mathbf{T}^{2}$-action.

The localization calculation is similar in flavor to the genus 0 Gromov-Witten calculation of $Q$ in [11. However, two new issues arise:

(i) The $\mathbf{T}^{2}$-action has fixed loci in $\widetilde{M}_{D}\left(\mathbb{C P}^{4} / \mathbb{C P}_{\mathbb{R}}^{4}, d\right)$ with moving images in $\mathbb{C P}^{4}$.

(ii) The equivariant restriction of $e\left(F_{d}\right)$ to the $\mathbf{T}^{2}$-fixed locus depends upon the orientation of $F_{d}$.

Issue (i) is handled by identifying the nonrigid contributions with the equivariant correlators $S_{Q}$ studied by Givental [5, 16. Issue (ii) requires an explicit evaluation of the signs occurring in the orientation. The derivation is presented in Section 4 .

The sum over $\mathbf{T}^{2}$-fixed point loci required for the localization formula is executed in two steps. Subsums with fixed intersection type with $\mathbb{C P}_{\mathbb{R}}^{4}$ are evaluated using Givental's equivariant mirror transformation for $S_{Q}$. Finally, the sum over intersection types is evaluated explicitly after appropriate equivariant specialization. The interaction of the orientation signs with the localization sum is an interesting aspect of the calculation. The outcome is a proof of Theorem 1.

3.2. $\mathbf{T}^{2}$ Torus-fixed disk maps. We first study the $\mathbf{T}^{2}$-fixed locus of the moduli space of stable disk maps. Let

$$
\left[f:(D, \partial D) \rightarrow\left(\mathbb{C P}^{4}, \mathbb{C P}_{\mathbb{R}}^{4}\right)\right] \in \bar{M}_{D}\left(\mathbb{C P}^{4} / \mathbb{C P}_{\mathbb{R}}^{4}, d\right)^{\mathbf{T}^{2}}
$$

be a $\mathbf{T}^{2}$-fixed map.

The boundary $\partial D$ distinguishes a minimal, $c$-invariant, central curve $P \subset C$ of the domain of the reflected map

$$
\left[\tilde{f}: C \rightarrow \mathbb{C P}^{4}\right] \in \bar{M}_{\mathbb{R}}\left(\mathbb{C P}^{4}, d\right)
$$

satisfying $\partial D=P_{\mathbb{R}}$. The central degree of $f$ is the degree of the restriction

$$
\tilde{f}_{P}: P \rightarrow \mathbb{C P}^{4} .
$$

The central degree $p$ is positive, odd, and bounded by $d$. The moduli point

$$
\left[\tilde{f}_{P}\right] \in \bar{M}_{\mathbb{C}}\left(\mathbb{C P}^{4}, p\right)
$$

is fixed for the full complexified action of $\left(\mathbb{C}^{*}\right)^{2}$ on $\mathbb{C P}^{4}$. 
Lemma 4. The two lines $L, L^{\prime} \subset \mathbb{C P}^{4}$ are the only $\left(\mathbb{C}^{*}\right)^{2}$-invariant curves of odd degree defined over $\mathbb{R}$ in $\mathbb{C P}^{4}$.

Proof. A real $\left(\mathbb{C}^{*}\right)^{2}$-invariant subcurve must lie in one of the two planes

$$
\begin{aligned}
& \left\{\left[z_{0}, z_{1}, z_{2}, 0,0\right] \mid z_{0}, z_{1}, z_{2} \in \mathbb{C}\right\}, \\
& \left\{\left[z_{0}, 0,0, z_{3}, z_{4}\right] \mid z_{0}, z_{3}, z_{4} \in \mathbb{C}\right\} .
\end{aligned}
$$

In the first case, $L$ is the only real $\left(\mathbb{C}^{*}\right)^{2}$-invariant line. Moreover, all nonlinear $\left(\mathbb{C}^{*}\right)^{2}$-orbits are of degree 2 . The argument in the second case is identical.

A node of $P_{\mathbb{R}}$ must map via $\tilde{f}$ to the unique real fixed point $\zeta_{0} \in \mathbb{C P}^{4}$. Since $\tilde{f}(P)$ must equal either $L$ or $L^{\prime}, \tilde{f}(P)$ cannot contain $\zeta_{0}$. Hence, $P_{\mathbb{R}}$ cannot contain a node. We obtain the following result.

Lemma 5. The central curve $P$ is $\mathbb{C P}^{1}$ and

$$
\tilde{f}_{P}: P \rightarrow L \text { or } L^{\prime}
$$

is a Galois cover of odd degree $p$.

The original disk map $f$ is obtained from one half of $\tilde{f}$. Hence one half of $P$ is selected by $D$. A half of $P$ determines a pair $(\zeta, p)$ where

$$
\zeta \in\left\{\zeta_{1}, \zeta_{2}, \zeta_{3}, \zeta_{4}\right\}
$$

is a nonreal fixed point and $p$ is the central degree.

The data $(\zeta, p)$ is the termed the intersection type of $f$ with the real Lagrangian $\mathbb{C P}_{\mathbb{R}}^{4} \subset \mathbb{C P}^{4}$. The half of $P$ selected by $D$ is the intersection disk.

While we have analyzed $\bar{M}_{D}\left(\mathbb{C P}^{4} / \mathbb{C P}_{\mathbb{R}}^{4}, d\right)^{\mathbf{T}^{2}}$, we are actually interested in $\widetilde{M}_{D}\left(\mathbb{C P}^{4} / \mathbb{C P}_{\mathbb{R}}^{4}, d\right)^{\mathbf{T}^{2}}$. If fact, we have proven

$$
\bar{M}_{D}\left(\mathbb{C P}^{4} / \mathbb{C P}_{\mathbb{R}}^{4}, d\right)^{\mathbf{T}^{2}}=\widetilde{M}_{D}\left(\mathbb{C P}^{4} / \mathbb{C P}_{\mathbb{R}}^{4}, d\right)^{\mathbf{T}^{2}}
$$

since the $\mathbf{T}^{2}$-fixed maps are not corner points of $\bar{M}_{D}\left(\mathbb{C P}^{4} / \mathbb{C P}_{\mathbb{R}}^{4}, d\right)$.

3.3. Intersection disk term. The localization calculation of

$$
N_{d}^{\text {disk }}=\int_{\widetilde{M}_{D}\left(\mathbb{C P}^{4} / \mathbb{C P}_{\mathbb{R}}^{4}, d\right)} e\left(F_{d}\right)
$$

is the sum over the contributions of the $\mathbf{T}^{2}$-fixed loci. We may separate the contributions by intersection type:

$$
N_{d}^{d i s k}=\sum_{i=1}^{4} \sum_{p \text { odd }} \operatorname{Cont}_{\left(\zeta_{i}, p\right)}\left(N_{d}^{d i s k}\right) .
$$

The intersection disk term $I\left(\zeta_{i}, p\right)$ of $\operatorname{Cont}_{\left(\zeta_{i}, p\right)}\left(N_{d}^{\text {disk }}\right)$ is the contribution of the unique $\mathbf{T}^{2}$-fixed map

$$
f:(D, \partial D) \rightarrow\left(\mathbb{C P}^{4}, \mathbb{C P}_{\mathbb{R}}^{4}\right)
$$

incident to $\zeta_{i}$ with central degree $p$ and domain consisting only of the intersection disk.

Define the rational function $C_{p}\left(\lambda, \lambda^{\prime}\right)$ of degree 0 by the following formula:

$$
C_{p}\left(\lambda, \lambda^{\prime}\right)=\frac{(-1)^{\frac{p-1}{2}}}{p} \frac{2 \lambda}{p} \frac{\frac{(5 p) ! !}{p ! p ! !}\left(\frac{\lambda}{2 p}\right)^{p}}{\prod_{i=0}^{(p-1) / 2}\left(\left(1-\frac{2 i}{p}\right) \lambda-\lambda^{\prime}\right)\left(\left(1-\frac{2 i}{p}\right) \lambda+\lambda^{\prime}\right)} .
$$


Lemma 6. For an appropriate choice of Spin structure on $Q_{\mathbb{R}}$, we have

$$
I\left(\zeta_{1}, p\right)=I\left(\zeta_{2}, p\right)=C_{p}\left(\lambda, \lambda^{\prime}\right), \quad I\left(\zeta_{3}, p\right)=I\left(\zeta_{4}, p\right)=C_{p}\left(\lambda^{\prime}, \lambda\right) .
$$

Changing the Spin structure changes the formulas by -1 for all $p$.

The proof of Lemma 6 is given in Section 4. The most interesting aspect is the calculation of the prefactor $(-1)^{\frac{p-1}{2}}$ obtained from the orientations of the moduli space $\bar{M}_{D}\left(\mathbb{C P}^{4} / \mathbb{C P}_{\mathbb{R}}^{4}, p\right)$ and the bundle $F_{p}$.

3.4. Givental's correlator $S_{Q}$. Let $\bar{M}_{0,2}\left(\mathbb{C P}^{4}, r\right)$ be the moduli space of 2-pointed stable complex algebraic maps to $\mathbb{C P}^{4}$ of genus 0 and degree $r$. Let

$$
e_{i}: \bar{M}_{0,2}\left(\mathbb{C P}^{4}, r\right) \rightarrow \mathbb{C P}^{4}
$$

be the evaluation at the $i^{\text {th }}$ marking, and let $\psi_{i}$ denote the Chern class of the $i^{\text {th }}$ cotangent line. Let

$$
E_{r} \rightarrow \bar{M}_{0,2}\left(\mathbb{C P}^{4}, r\right)
$$

be the complex vector bundle with fiber

$$
\left.E_{r}\right|_{\left[f: C \rightarrow \mathbb{C P}^{4}\right]}=H^{0}\left(C, \tilde{f}^{*} \mathcal{O}_{\mathbb{C P}^{4}}(5)\right)_{\mathbb{C}} .
$$

Following the notation of Section 2.2 of [16], Givental's equivariant correlator $S_{Q}$ for the torus $\mathbf{T}^{2}$ is defined by

$$
S_{Q}(T, \hbar)=\frac{1}{5 H} \sum_{r \geq 0} e^{(H / \hbar+r) T} e_{2 *}\left(\frac{c_{\text {top }}\left(E_{r}\right)}{\hbar-\psi_{2}}\right) \in H_{\mathbf{T}^{2}}^{*}\left(\mathbb{C P}^{4}\right),
$$

where $H$ is the hyperplane class,

$$
H=c_{1}\left(\mathcal{O}_{\mathbb{C P}^{4}}(1)\right) \in H_{\mathbf{T}^{2}}^{*}\left(\mathbb{C P}^{4}\right) .
$$

The sum in (8) is over all nonnegative integers $r$. The unstable degree 0 term is defined by

$$
\frac{1}{5 H} e_{2 *}\left(\frac{c_{\text {top }}\left(E_{0}\right)}{\hbar-\psi_{2}}\right)=1
$$

Let $\left[\zeta_{i}\right] \in H_{\mathbf{T}^{2}}^{*}\left(\mathbb{C P}^{4}\right)$ denote the Poincare dual of the class of the fixed point. For classes $\mu, \nu \in H_{\mathbf{T}^{5}}^{*}\left(\mathbb{C P}^{4}\right)$, let

$$
\langle\mu, \nu\rangle \in \mathbb{Q}\left[\lambda, \lambda^{\prime}\right]
$$

denote the equivariant intersection pairing. For example,

$$
\left\langle H,\left[\zeta_{i}\right]\right\rangle=\lambda_{i},
$$

following convention (7).

The intersection pair of the equivariant correlator will arise in the localization analysis:

$$
\begin{aligned}
\left\langle S_{Q}(T, \hbar),\left[\zeta_{i}\right]\right\rangle & =\frac{1}{5 \lambda_{i}} \sum_{r \geq 0} e^{\left(\lambda_{i} / \hbar+r\right) T} \int_{\bar{M}_{0,2}\left(\mathbb{C P}^{4}, r\right)} \frac{c_{\text {top }}\left(E_{r}\right)}{\hbar-\psi_{2}} e_{2}^{*}\left(\left[\zeta_{i}\right]\right) \\
& =\frac{\hbar^{-1}}{5 \lambda_{i}} \sum_{r \geq 0} e^{\left(\lambda_{i} / \hbar+r\right) T} \int_{\bar{M}_{0,1}\left(\mathbb{C P}^{4}, r\right)} \frac{c_{\text {top }}\left(E_{r}\right)}{\hbar-\psi_{1}} e_{1}^{*}\left(\left[\zeta_{i}\right]\right)
\end{aligned}
$$

where the string equation is used in the second line. In degree 0 , the unstable 1 -pointed term is defined by the second equality. 
3.5. Contributions of type $\left(\zeta_{i}, p\right)$. The $\mathbf{T}^{2}$-fixed loci of the moduli space $\widetilde{M}_{D}\left(\mathbb{C P}^{4} / \mathbb{C P}_{\mathbb{R}}^{4}, d\right)$ of type $\left(\zeta_{i}, p\right)$ may be quite complicated. However, every map

$$
[f] \in \widetilde{M}_{D}\left(\mathbb{C P}^{4} / \mathbb{C P}_{\mathbb{R}}^{4}, d\right)^{\mathbf{T}^{2}}
$$

of type $\left(\zeta_{i}, p\right)$ ends in the same intersection disk. By expanding the localization formula, the intersection disk term $I\left(\zeta_{i}, p\right)$ can be factored out of $\operatorname{Cont}_{\left(\zeta_{i}, p\right)}\left(N_{d}^{\text {disk }}\right)$ by removing the intersection disk from $f$.

What remains after the intersection disk is removed from $f$ ? In fact, every genus 0 stable complex map

$$
\left[f^{\prime}\right] \in e_{1}^{-1}\left(\zeta_{i}\right) \subset \bar{M}_{0,1}\left(\mathbb{C P}^{4}, r\right)^{\left(\mathbb{C}^{*}\right)^{2}}
$$

can be found. The stable disk map $f$ is obtained by attaching the $\left(\zeta_{i}, p\right)$-intersection disk to $f^{\prime}$ at the marking.

A direct unraveling of the localization formulas yields the following fundamental result. Let

$$
\operatorname{Cont}_{\left(\zeta_{i}, p\right)}\left(\mathcal{F}^{d i s k}\right)=\sum_{d \text { odd }} e^{d T / 2} \operatorname{Cont}_{\left(\zeta_{i}, p\right)}\left(N_{d}^{d i s k}\right) .
$$

Lemma 7. We have

$$
\operatorname{Cont}_{\left(\zeta_{i}, p\right)}\left(\mathcal{F}^{d i s k}\right)=\left\langle S_{Q}\left(T, \frac{2}{p} \lambda_{i}\right),\left[\zeta_{i}\right]\right\rangle \cdot I\left(\zeta_{i}, p\right)
$$

for $1 \leq i \leq 4$.

Proof. The sum on left side can be indexed more conveniently as

$$
\operatorname{Cont}_{\left(\zeta_{i}, p\right)}\left(\mathcal{F}^{\text {disk }}\right)=\sum_{r \geq 0} e^{\left(\frac{p}{2}+r\right) T} \operatorname{Cont}_{\left(\zeta_{i}, p\right)}\left(N_{p+2 r}^{\text {disk }}\right) .
$$

The right side of the equality may be expanded as

$$
\sum_{r \geq 0} e^{\left(\frac{p}{2}+r\right) T} \int_{\bar{M}_{0,1}\left(\mathbb{C P}^{4}, r\right)} \frac{c_{\mathrm{top}}\left(E_{r}\right)}{\frac{2}{p} \lambda_{i}-\psi_{1}} e_{1}^{*}\left(\left[\zeta_{i}\right]\right) \cdot \frac{I\left(\zeta_{i}, p\right)}{\left(5 \lambda_{i}\right)\left(\frac{2}{p} \lambda_{i}\right)} .
$$

The lemma is obtained from the equality

$$
\operatorname{Cont}_{\left(\zeta_{i}, p\right)}\left(N_{p+2 r}^{d i s k}\right)=\int_{\bar{M}_{0,1}\left(\mathbb{C P}^{4}, r\right)} \frac{c_{\mathrm{top}}\left(E_{r}\right)}{\frac{2}{p} \lambda_{i}-\psi_{1}} e_{1}^{*}\left(\left[\zeta_{i}\right]\right) \cdot \frac{I\left(\zeta_{i}, p\right)}{\left(5 \lambda_{i}\right)\left(\frac{2}{p} \lambda_{i}\right)} .
$$

To prove (9), we apply $\mathbf{T}^{2}$-localization to the integral on the right. We do not fully expand the $\mathbf{T}^{2}$-localization formula. As was previously discussed, understanding the geometry of the individual $\mathbf{T}^{2}$-fixed loci is difficult, as there are positive-dimensional families of $\mathbf{T}^{2}$-fixed curves. However, (9) has a much simpler proof. Since both sides are expressed as $\mathbf{T}^{2}$-residue integrals by localization, we need only match the geometries.

First, the $\mathbf{T}^{2}$-fixed loci of the two sides of (9) are in bijective correspondence. Given a $\mathbf{T}^{2}$-fixed locus on the right, the addition of the intersection disk $I\left(\zeta_{i}, p\right)$ at the marking 1 yields a $\mathbf{T}^{2}$-fixed locus of the right side. The reverse direction is obtained by stripping the intersection disk.

Second, since the $\mathbf{T}^{2}$-fixed loci on the left and right are both nonsingular, the correspondence induces an isomorphism of $\mathbf{T}^{2}$-fixed loci up to the automorphism factor of the intersection disk.

Finally, we must match the $\mathbf{T}^{2}$-fixed obstruction theories. Let

$$
\left[f_{D}: D=C \cup I\left(\zeta_{i}, p\right) \rightarrow \mathbb{C P}^{4}\right] \in \widetilde{M}_{D}\left(\mathbb{C P}^{4} / \mathbb{C P}_{\mathbb{R}}^{4}, p+2 r\right)^{\mathbf{T}^{2}}
$$


be a map with

$$
\left[f_{C}: C \rightarrow \mathbb{C P}^{4}\right] \in \operatorname{ev}_{1}^{-1}\left(\zeta_{i}\right) \subset \bar{M}_{0,1}\left(\mathbb{C P}^{4}, r\right)^{\mathbf{T}^{2}}
$$

By the normalization sequence,

$$
\left.\left.\left.\left.0 \rightarrow F_{p+2 r}\right|_{\left[f_{D}\right]} \rightarrow E_{r}\right|_{\left[f_{C}\right]} \oplus F_{p}\right|_{\left[I\left(\zeta_{i}, p\right)\right]} \rightarrow \mathcal{O}_{\mathbb{C P}^{4}}(5)\right|_{\zeta_{i}} \rightarrow 0 .
$$

Hence, the numerator in the residue integral on the left of (9) is

$$
e\left(F_{p+2 r}\right)=c_{\mathrm{top}}\left(E_{r}\right) \cdot \frac{e\left(F_{p}\right)}{5 \lambda_{i}} .
$$

Similarly, the denominator of the residue integral on the left of (9) is

$$
\frac{1}{e\left(\operatorname{Nor}_{\left[f_{D}\right]}\right)}=\frac{1}{c_{\text {top }}\left(\operatorname{Nor}_{\left[f_{C}\right]}\right)} \frac{c_{\text {top }}\left(\operatorname{Tan}_{\zeta_{i}}\right)}{\left(\frac{2}{p} \lambda_{i}-\psi_{1}\right)\left(\frac{2}{p} \lambda_{i}\right)} \frac{1}{e\left(\operatorname{Nor}_{\left[I\left(\zeta_{i}, p\right)\right]}\right)} \text {. }
$$

The middle terms are obtained from tangent bundle, node smoothing, and automorphism factors. Putting (10) and (11) together, we obtain the exact matching needed for (9).

The factorization of (9) properly reflects the orientation on the moduli space $\widetilde{M}_{D}\left(\mathbb{C P}^{4} / \mathbb{C P}_{\mathbb{R}}^{4}, d\right)$. The orientation factorization is easily obtained from [24].

Such arguments form the geometric basis of [5, 16]. Though the $\mathbf{T}^{2}$-action on $\widetilde{M}_{D}\left(\mathbb{C P}^{4} / \mathbb{C P}_{\mathbb{R}}^{4}, d\right)$ has fixed loci corresponding to moving maps, the issue is completely avoided by the lemma.

3.6. Mirror transforms. We review the mirror transforms relating $S_{Q}(T, \hbar)$ to

$$
S_{Q}^{*}(t, \hbar)=\frac{1}{5 H} \sum_{r \geq 0} e^{\left(\frac{H}{\hbar}+r\right) t} \frac{\Pi_{s=0}^{5 r}(5 H+s \hbar)}{\Pi_{j=0}^{4} \Pi_{s=1}^{r}\left(H-\lambda_{j}+s \hbar\right)}
$$

following Section 4.4 of 16 . Let

The mirror map $T(t)=I_{1} / I_{0}(t)$ discussed in Section 0.1 can be written explicitly.

$$
F(q)=\sum_{r=0}^{\infty} q^{r} \frac{(5 r) !}{(r !)^{5}}, \quad G_{l}(q)=\sum_{r=1}^{\infty} q^{r} \frac{(5 r) !}{(r !)^{5}}\left(\sum_{s=1}^{l r} \frac{1}{s}\right)
$$

Then

$$
T=t+\frac{5\left(G_{5}\left(e^{t}\right)-G_{1}\left(e^{t}\right)\right)}{F\left(e^{t}\right)}
$$

is the mirror map. Exponentiating yields

$$
\exp (T)=\exp (t) \cdot \exp \left(\frac{5\left(G_{5}\left(e^{t}\right)-G_{1}\left(e^{t}\right)\right)}{F\left(e^{t}\right)}\right) .
$$

The equivariant mirror transformation for the torus $\mathbf{T}^{2}$ is

$$
S_{Q}(T(t), \hbar)=\frac{1}{F\left(e^{t}\right)} S_{Q}^{*}(t, \hbar) .
$$

Transforms for the equivariant pairings are a direct consequence,

$$
\left\langle S_{Q}\left(T(t), \frac{2}{p} \lambda_{i}\right),\left[\zeta_{i}\right]\right\rangle=\frac{1}{F\left(e^{t}\right)}\left\langle S_{Q}^{*}\left(t, \frac{2}{p} \lambda_{i}\right),\left[\zeta_{i}\right]\right\rangle .
$$


3.7. Theorem 1. We now complete the calculation of $\mathcal{F}^{\text {disk }}$,

$$
\begin{aligned}
\mathcal{F}^{\text {disk }} & =\sum_{i=1}^{4} \sum_{p \text { odd }} \operatorname{Cont}_{\left(\zeta_{i}, p\right)}\left(\mathcal{F}^{\text {disk }}\right) \\
& =\sum_{i=1}^{4} \sum_{p \text { odd }}\left\langle S_{Q}\left(T, \frac{2}{p} \lambda_{i}\right),\left[\zeta_{i}\right]\right\rangle \cdot I\left(\zeta_{i}, p\right) \\
& =\sum_{i=1}^{4} \sum_{p \text { odd }} \frac{1}{F\left(e^{t}\right)}\left\langle S_{Q}^{*}\left(t, \frac{2}{p} \lambda_{i}\right),\left[\zeta_{i}\right]\right\rangle \cdot I\left(\zeta_{i}, p\right) .
\end{aligned}
$$

The $i=1,2$ summands of the last line together yield

$$
\frac{4}{F\left(e^{t}\right)} \sum_{r \geq 0} \sum_{p \text { odd }} e^{\left(\frac{p}{2}+r\right) t} \frac{(-1)^{\frac{p-1}{2}}}{r !(p+r) ! p} \frac{2^{-(p+2 r)} \frac{(5 p+10 r) ! !}{(p+2 r) ! !}}{\prod_{1 \leq i \text { odd } \leq p+2 r}(i-p x)(i+p x)}
$$

written in terms of the homogeneous variable

$$
x=\frac{\lambda^{\prime}}{\lambda} \text {. }
$$

Similarly, the $i=3,4$ summands together yield

$$
\frac{4}{F\left(e^{t}\right)} \sum_{r \geq 0} \sum_{p \text { odd }} e^{\left(\frac{p}{2}+r\right) t} \frac{(-1)^{\frac{p-1}{2}}}{r !(p+r) ! p} \frac{2^{-(p+2 r)} \frac{(5 p+10 r) ! !}{(p+2 r) ! !}}{\prod_{1 \leq i \text { odd } \leq p+2 r}\left(i-p x^{-1}\right)\left(i+p x^{-1}\right)} .
$$

The final step is to observe that the localization calculation of $\mathcal{F}^{\text {disk }}$ is a weight independent global integral. Hence, we may evaluate the summation in the $x \rightarrow 0$ limit. Only the $i=1,2$ terms survive the limit:

$$
\mathcal{F}^{\text {disk }}=\frac{4}{F\left(e^{t}\right)} \sum_{r \geq 0} \sum_{p \text { odd }} e^{\left(\frac{p}{2}+r\right) t} \frac{(-1)^{\frac{p-1}{2}}}{r !(p+r) ! p} \frac{2^{-(p+2 r)} \frac{(5 p+10 r) ! !}{(p+2 r) ! !}}{(p+2 r) ! !(p+2 r) ! !} .
$$

The identity for odd $d$,

$$
\sum_{1 \leq p \text { odd } \leq d} \frac{(-1)^{\frac{p-1}{2}}}{\left(\frac{d-p}{2}\right) !\left(\frac{d-p}{2}+p\right) ! p}=\frac{2^{d-1}}{(d ! !)^{2}},
$$

restated in Lemma 8 below concludes the proof of Theorem 1 ,

$$
\mathcal{F}^{\text {disk }}(T)=\frac{2}{F\left(e^{t}\right)} \sum_{d \text { odd }} e^{d t / 2} \frac{(5 d) ! !}{(d ! !)^{5}} .
$$

After regrouping the factors and reindexing the sum, the identity (12) is equivalent to the following result.

Lemma 8. For d odd,

$$
\sum_{k=0}^{d}\left(\begin{array}{l}
d \\
k
\end{array}\right)(-1)^{k} \frac{d}{d-2 k}=(-1)^{\frac{d-1}{2}} \frac{2^{2 d-1}}{\left(\begin{array}{l}
d-1 \\
\frac{d-1}{2}
\end{array}\right)} .
$$

Proof. An elementary derivation is left to the reader. A geometric proof is obtained from the multiple cover calculations in Section 6 


\section{INTERSECTION DISK TERMS}

4.1. Overview. We now derive the signs needed in the localization calculation and prove Lemma 6. The orientations of the moduli spaces and vector bundles used to calculate $N_{d}^{\text {disk }}$ arise from the natural orientation of the determinant of the Cauchy-Riemann Pin boundary value problem developed in 19. Briefly, a Cauchy-Riemann boundary value problem consists of a topological complex vector bundle $E$ over a Riemann surface with boundary $\Sigma$, a totally real sub-bundle $F$ over the boundary $\partial \Sigma$, and a generalized Cauchy-Riemann operator $d^{\prime \prime}$ on $E$. Given a $P$ in structure on $F$, and a choice of orientation on $F$ if orientable, one may define a canonical orientation of the determinant

$$
\operatorname{det}\left(d^{\prime \prime}\right):=\Lambda^{\max }\left(\operatorname{ker} d^{\prime \prime}\right) \otimes \Lambda^{\max }\left(\operatorname{coker} d^{\prime \prime}\right)^{*} .
$$

Reversing the Pin structure on $F$ reverses the canonical orientation [19, Lemma $2.10]$.

For the calculations of weights below, we will only be concerned with the situation where $E \rightarrow D$ is the restriction of an algebraic vector bundle $\tilde{E} \rightarrow \mathbb{C P}^{1}$ defined over $\mathbb{R}$. We take $F=\tilde{E}_{\mathbb{R}}$, and we take $d^{\prime \prime}$ to be the restriction to $D$ of the $\bar{\partial}$ operator defined by the holomorphic structure on $\tilde{E}$. The identifications

$$
\operatorname{ker}\left(d^{\prime \prime}\right)=H^{0}\left(\mathbb{C P}^{1}, \tilde{E}\right)_{\mathbb{R}}, \quad \operatorname{coker}\left(d^{\prime \prime}\right)=H^{1}\left(\mathbb{C P}^{1}, \tilde{E}\right)_{\mathbb{R}}
$$

are easily obtained. So, an orientation of $\operatorname{det}\left(d^{\prime \prime}\right)$ gives an orientation of the virtual vector space

$$
H^{0}\left(\mathbb{C P}^{1}, \tilde{E}\right)_{\mathbb{R}}-H^{1}\left(\mathbb{C P}^{1}, \tilde{E}\right)_{\mathbb{R}} .
$$

Note, however, the orientation depends on the choice of

$$
D \subset \mathbb{C P}^{1} \backslash \mathbb{C P}_{\mathbb{R}}^{1}
$$

So, we cannot entirely forget the origins of our orientation in a boundary value problem. For convenience, we introduce the notation

$$
H^{0}(D, \tilde{E}):=\operatorname{ker}\left(d^{\prime \prime}\right), \quad H^{1}(D, \tilde{E}):=\operatorname{coker}\left(d^{\prime \prime}\right) .
$$

Section 4.2 interprets the symplectic geometric definition of the orientation algebraically. Section 4.3 calculates the localization contributions from a fixed point of the torus action using the combinatorics of exact sequences and the formula for the tensor product of real representations of $S^{1}$. The algebraically inclined reader may safely skip all of Section 4.2 besides the statement of Lemma 9.

4.2. Weights of sections of a line bundle. Let $\mathbf{T}^{n}$ denote the real $n$-dimensional torus and let $\mathfrak{t}^{n}$ denote its Lie algebra. A weight is a homomorphism of real vector spaces from $\mathfrak{t}^{n}$ to $\mathbb{C}$. Let $V$ be a 2 -dimensional real irreducible oriented representation of $\mathbf{T}^{n}$ and let $\rho$ be the associated homomorphism

$$
\rho: \mathbf{T}^{n} \rightarrow \mathbf{G L}(V) .
$$

Let $h: V \rightarrow \mathbb{C}$ be an orientation preserving real linear homomorphism such that the associated homorphism

$$
\tilde{h}: \mathbf{G L}(V) \rightarrow \mathbf{G L}(\mathbb{C})
$$

satisfies

$$
\operatorname{Im}(\tilde{h} \circ \rho) \subset \operatorname{Aut}_{\mathbb{C}}(\mathbb{C}) \simeq \mathbb{C}^{\times} .
$$

Note that $h$ is defined by these conditions up to homothety and hence $\tilde{h}$ is unique. Differentiating $\tilde{h} \circ \rho$ we obtain the weight of $V$. 
We denote by $V_{\lambda}$ the 2-dimensional real oriented representation of $\mathbf{T}^{n}$ of weight $\lambda$, where $\lambda$ may be fractional. If $\lambda=0$ we denote by $V_{\lambda}$ the trivial 2-dimensional representation. Suppose $V$ is an oriented real representation of $\mathbf{T}^{n}$ isomorphic to $V_{\lambda}$. A priori, $V$ consists of two data: a $\mathbf{T}^{n}$-action on the vector space $V$ and an orientation of the vector space $V$. However, except in the case $\lambda=0$, the $\mathbf{T}^{n}$-action on $V$ and the knowledge that $V \simeq V_{\lambda}$ determine the orientation of $V$. Indeed, if $\lambda \neq 0$, there is a unique up to homothety $\mathbf{T}^{n}$-equivariant isomorphism

$$
i: V \stackrel{\sim}{\rightarrow} V_{\lambda} .
$$

The orientation on $V$ must agree with the orientation induced by $i$.

Let $m$ be a positive odd integer. From [19], in order to define the canonical orientation of $\operatorname{det}\left(d^{\prime \prime}\right)$ mentioned above, a $\operatorname{Pin}$ structure $\mathfrak{p}_{-1}$ on $\mathcal{O}(-1)_{\mathbb{R}} \rightarrow \partial D$ must be chosen. We fix such a $\operatorname{Pin}$ structure. Set $W_{\lambda}=V_{\lambda} \otimes \mathbb{C}$. Choosing a connected component of the complement of

$$
\mathbb{C P}_{\mathbb{R}}^{1} \subset \mathbb{C P}^{1}=\mathbb{C P}\left(W_{\lambda}\right)
$$

is equivalent to choosing an orientation on $V_{\lambda}$. The action of $\mathbf{T}^{n}$ on $W_{\lambda}$ naturally induces an action on $H^{0}(D, \mathcal{O}(m))$ when we think of $D$ as the disk that induces the orientation of $V_{\lambda}$. Indeed, as not oriented vector spaces, clearly

$$
H^{0}(D, \mathcal{O}(m)) \simeq \operatorname{Sym}^{m}\left(V_{\lambda}^{*}\right) .
$$

We only consider the $m$ odd case.

The main goal of this section is to prove the following lemma which examines when isomorphism (13) preserves orientation. Equip the vector space $H^{0}(D, \mathcal{O}(m))$ with the $\mathbf{T}^{n}$-action induced from $V_{-\lambda}$. Let $\mathfrak{p}$ be a Pin structure on $\mathcal{O}(m)_{\mathbb{R}} \rightarrow \partial D$. Since $m$ is odd, $\mathcal{O}(m)_{\mathbb{R}} \simeq \mathcal{O}(-1)_{\mathbb{R}}$ as real topological vector bundles.

Lemma 9. Assume that $\mathfrak{p}$ agrees with $\mathfrak{p}_{-1}$. With respect to the canonical orientation induced by $\mathfrak{p}$,

$$
H^{0}(D, \mathcal{O}(m)) \simeq \bigoplus_{i=0}^{(m-1) / 2} V_{(2 i+1) \lambda}
$$

Proof. First, the canonical orientation induced by $\mathfrak{p}$ can be expressed as the complex orientation induced by an explicit complex structure on $H^{0}(D, \mathcal{O}(m))$. Indeed, by gluing sections, we have an exact sequence

$$
\begin{aligned}
0 \rightarrow H^{0} & (D, \mathcal{O}(m)) \\
& \stackrel{g^{-1}}{\rightarrow} H^{0}(D, \mathcal{O}(-1)) \oplus H^{0}\left(\mathbb{C P}^{1}, \mathcal{O}((m+1) / 2)\right) \stackrel{h}{\rightarrow} \mathbb{C} \rightarrow 0 .
\end{aligned}
$$

By definition [19, Proposition 2.8], after equipping the latter two terms of the sequence with the complex orientation, the sequence induces the desired orientation on the first term. Note that $g^{-1}$, the inverse of the gluing map, is not canonical, but the set of all choices is connected. So, the induced orientation is well-defined. To calculate the orientation, we may fix a particular choice of the gluing map and calculate the induced complex structure on $H^{0}(D, \mathcal{O}(m))$.

We digress for a moment to explain the relationship between different possible descriptions of $H^{0}(D, \mathcal{O}(m))$. Let $w_{0}, w_{1}$ be standard linear coordinates on $W_{\lambda}$ such 
that $w_{0}, w_{1}$ are real precisely on the real locus of $W_{\lambda}$. Then, $H^{0}(D, \mathcal{O}(m))$ may be identified with the vector space of homogeneous polynomials

$$
p\left(w_{0}, w_{1}\right)=\sum_{i=0}^{m} a_{i} w_{0}^{d-j} w_{1}^{j}, \quad a_{i} \in \mathbb{R} .
$$

Now, we choose new coordinates $z_{0}, z_{1}$, such that

$$
\begin{array}{ll}
z_{0}=w_{0}-i w_{1}, & z_{1}=w_{0}+i w_{1}, \\
w_{0}=\frac{z_{0}+z_{1}}{2}, & w_{1}=i \frac{z_{0}-z_{1}}{2} .
\end{array}
$$

Then, since $p\left(z_{0}, z_{1}\right)=\overline{p\left(\bar{z}_{1}, \bar{z}_{0}\right)}$, we have

$$
p\left(z_{0}, z_{1}\right)=\sum_{j=0}^{m} b_{i} z_{0}^{d-j} z_{1}^{j}, \quad b_{j}=\bar{b}_{d-j} .
$$

On the other hand, by definition, $H^{0}(D, \mathcal{O}(m))$ is the space of the solutions $\xi$ of the Cauchy Riemann equations on unit disk

$$
D=\{|z| \leq 1\} \subset \mathbb{C}
$$

satisfying the totally real boundary conditions

$$
\xi(z) \in \mathbb{R} z^{m / 2}, \quad|z|=1 .
$$

In the future, we refer to these boundary conditions as $L(m)$. Expanding in power series about $z=0$, it is not hard to see that the boundary conditions imply

$$
\xi(z)=\sum_{j=0}^{m} b_{i} z^{i}, \quad b_{j}=\bar{b}_{d-j}
$$

So, solutions $\xi$ arise naturally from homogeneous polynomials $p\left(z_{0}, z_{1}\right)$ by trivializing $\mathcal{O}(m)$ over $\left\{z_{0} \neq 0\right\}$ by the section $z_{0}^{m}$ and making the identification $z=z_{1} / z_{0}$.

For the following argument, we view sections $\xi \in H^{0}(D, \mathcal{O}(m))$ as in (16). Referring to the exact sequence (15), we construct a gluing map

$$
g: \operatorname{ker}(h) \rightarrow H^{0}(D, \mathcal{O}(m))
$$

as follows. Suppose we identify the point at which we glue sections with $\infty \in \mathbb{C P}^{1}$. Then, by taking the standard trivialization of the sheaf $\mathcal{O}((m+1) / 2)$ over the standard coordinate chart on $\mathbb{C P}^{1}$ centered at 0 , we may identify $\operatorname{ker}(h)$ with the set of polynomials $q(z)$ of degree less than or equal to $(m-1) / 2$. Let

$$
\beta: D \rightarrow \mathbb{R}
$$

be a cutoff function depending only on $|z|$ such that $\beta(0)=1$ and $\beta(z)=0$ for $|z|>1 / 2$. Given a polynomial $q$, we define a pre-gluing by

$$
\tilde{q}(z)=\beta(z) q(z) .
$$

Let

$$
P: C^{\infty}((D, \partial D),(\mathbb{C}, L(m))) \rightarrow H^{0}(D, \mathcal{O}(m))
$$

denote the $L^{2}$ projection. We define $g$ by

$$
g(q)=P \tilde{q} .
$$

Due to our choice of gluing map, it is easy to calculate that

$$
g\left(b z^{j}\right)=\bar{b} z^{m-j}+b z^{j} .
$$


Let $\xi$ be as in (16) and let $I$ denote the complex structure that $g$ induces on $H^{0}(D, \mathcal{O}(m))$. It follows that

$$
(I \xi)(z)=i \sum_{j=0}^{(m-1) / 2} b_{j} z^{j}-b_{m-j} z^{m-j} .
$$

Finally, we compute the weights of $H^{0}(D, \mathcal{O}(m))$. Since, by the definition of the action on $H^{0}(D, \mathcal{O}(m))$, we have identified $D$ with the hemisphere of $\mathbb{C P}^{1}$ inducing the intrinsic orientation of $V_{\lambda}$, we must have that $z$ transforms by $z \mapsto e^{-2 \lambda} z$.

Since $z=\frac{z_{1}}{z_{0}}$, it follows that in coordinates $z_{0}, z_{1}$, the action of $\mathbf{T}^{n}$ takes the form

$$
z_{0} \mapsto e^{i \lambda} z_{0}, \quad z_{1} \mapsto e^{-i \lambda} z_{1} .
$$

In other words, $\mathbf{T}^{n}$ acts by $e^{I(m-2 j) \lambda}$ on the section

$$
z_{0}^{j} z_{1}^{m-j}+z_{0}^{m-j} z_{1}^{j} \in H^{0}(D, \mathcal{O}(m)) .
$$

The decomposition (14) follows.

4.3. Localization contribution of an isolated fixed point. Let $X$ and $Y$ be two oriented real vector spaces, and let $e_{i} \in X$ and $f_{j} \in Y$ be oriented bases. There are different ways the tensor product $X \otimes Y$ can inherent an orientation from $X$ and $Y$. We use the right-to-left lexicographical ordering convention. We take

$$
e_{1} \otimes f_{1}, e_{2} \otimes f_{1}, \ldots, e_{1} \otimes f_{2}, e_{2} \otimes f_{2}, \ldots
$$

as an oriented basis of $X \otimes Y$.

If $X$ is even dimensional, then the orientation of $X \otimes Y$ is independent of the orientation of $Y$, and vice versa.

As before, let $V_{\lambda}$ be the 2-dimensional real representation of $\mathbf{T}^{n}$ with weight $\lambda$. The following result is a straightforward linear algebra calculation.

Lemma 10. With respect to the right-to-left lexicographical orientation of the tensor product,

$$
V_{\alpha} \otimes V_{\beta}=V_{\alpha+\beta} \oplus V_{\alpha-\beta} .
$$

4.4. Proof of Lemma 6 . Let

$$
\left[f:(D, \partial D) \rightarrow\left(\mathbb{C P}^{4}, \mathbb{C P}_{\mathbb{R}}^{4}\right)\right] \in \bar{M}_{D}\left(\mathbb{C P}^{4} / \mathbb{C P}_{\mathbb{R}}^{4}, d\right)
$$

denote a $\mathbf{T}^{2}$-fixed disk of type $\left(\zeta_{1}, p\right)$. After permuting indices, the proof given below applies to the other possible intersection types as well.

First, we will calculate the equivariant Euler class of the tangent space to $\bar{M}_{D}\left(\mathbb{C P}^{4} / \mathbb{C P}_{\mathbb{R}}^{4}, d\right)$ at $[f]$ which we denote $N_{f}$. We use the deformation exact sequence

$$
0 \rightarrow \operatorname{Aut}(D) \rightarrow \operatorname{Def}(f) \rightarrow \operatorname{Def}(D, f) \rightarrow 0 .
$$

Here, $\operatorname{Def}(f)$ denotes the space of first-order deformations of the map $f$, and $\operatorname{Def}(f, D)$ denotes the first-order deformations of $f$ modulo reparametrization the tangent space to $\bar{M}_{D}\left(\mathbb{C P}^{4} / \mathbb{C P}_{\mathbb{R}}^{4}, d\right)$ at $[f]$. To carry out the corresponding closed calculation, the weights of each of the first two terms are computed and divided with cancelling 0 -weights. In the open case, more information is needed about the weight 0 components since isomorphisms of two copies of the trivial real representation of $\mathbf{T}^{n}$ need not preserve orientation. We will use the real Euler sequence to linearize the exact sequence (18) to get a better handle on the sign. 


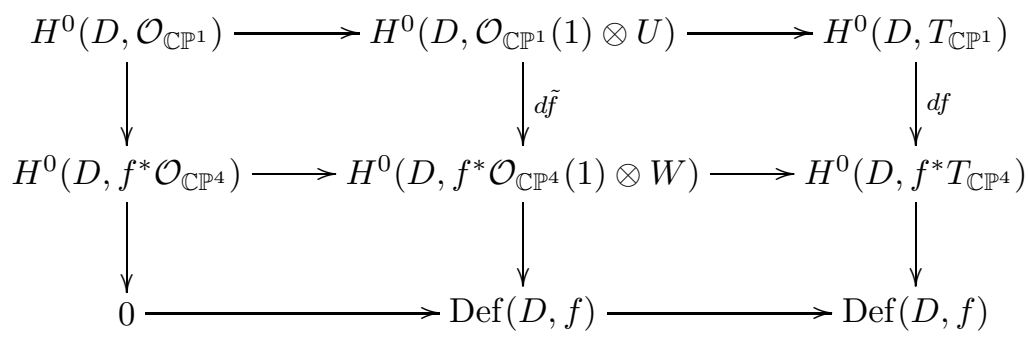

FiguRE 1

Let $X=V_{\lambda_{1} / p}$ and $Y=\mathbb{R} \oplus V_{\lambda_{1}} \oplus V_{\lambda_{3}}$. Let

$$
U=X \otimes \mathbb{C}, \quad W=Y \otimes \mathbb{C} .
$$

Let $Y^{\prime} \subset Y$ denote the 2-dimensional linear subspace corresponding to $V_{\lambda_{1}}$ and let $Y^{\prime \prime}$ denote the $\mathbf{T}^{n}$-invariant complement. Consider the commutative diagram in Figure 1. All rows and columns are exact. The rows are the sections functor applied to the Euler sequence. The rightmost column is obtained from the deformation exact sequence by the identifications

$$
\operatorname{Aut}(D)=H^{0}\left(D, T_{\mathbb{C P}^{1}}\right), \quad \operatorname{Def}(f)=H^{0}\left(D, f^{*} T_{\mathbb{C P}^{4}}\right) .
$$

In order to discuss the orientations of the vector spaces in Figure 1 we must digress for a moment on the subject of Pin structures. In the following, $\mathfrak{p}$ denotes a Pin structure on $\mathbb{C P}_{\mathbb{R}}^{4}, \mathfrak{p}^{\prime}$ denotes a Pin structure on $\mathcal{O}_{\mathbb{C P}^{4}}(5)_{\mathbb{R}}$ and $\mathfrak{s}$ denotes a Spin structure on $Q_{\mathbb{R}}$. To define $N_{d}^{\text {disk }}$, a structure $\mathfrak{s}$ was fixed. By Lemma 12 of Section 5, any two of $\mathfrak{p}, \mathfrak{p}^{\prime}, \mathfrak{s}$ naturally determine the third. As explained in the proof of Lemma 13 of Section 5 , the choice of $\mathfrak{p}$ and $\mathfrak{p}^{\prime}$ compatible with $\mathfrak{s}$ induces the isomorphism $\operatorname{det}\left(\mathcal{F}_{d}\right) \simeq \mathcal{L}$ used to determine the sign of $e\left(F_{d}\right)$. In particular, if we orient $N_{f}$ and $\left(F_{d}\right)_{f}$ using $\mathfrak{p}$ and $\mathfrak{p}^{\prime}$ compatible with $\mathfrak{s}$, we will be calculating the weights of the $\mathbf{T}^{2}$-action correctly.

In order to facilitate calculations, we choose $\mathfrak{p}$ in a convenient way. Then we let $\mathfrak{s}$ and $\mathfrak{p}$ induce $\mathfrak{p}^{\prime}$. Indeed, first choose a Pin structure $\hat{\mathfrak{p}}$ on $\mathcal{O}_{\mathbb{C P}^{4}}(1)_{\mathbb{R}}$. Via the natural homomorphism,

$$
\operatorname{Pin}(1) \rightarrow \operatorname{Pin}(5),
$$

induce a Pin structure $\tilde{\mathfrak{p}}$ on $\mathcal{O}_{\mathbb{C P}^{4}}(1)_{\mathbb{R}} \otimes Y$. In addition, equip $\mathcal{O}_{\mathbb{C P}_{\mathbb{R}}^{4}}$ with the canonical Spin structure. By [19, Lemma 8.1], via the Euler sequence, $\tilde{\mathfrak{p}}$ induces a Pin structure $\mathfrak{p}$ on $T_{\mathbb{C P}_{\mathbb{R}}^{4}}$. Since we have chosen Pin structures compatibly, by [19, Lemma 8.4], the middle row of the diagram in Figure 1 respects orientation. The columns respect orientation by definition.

At this point, we focus our attention on the middle column of Figure 1, which is the desired linearization of the deformation exact sequence. Because of the way we have induced $\tilde{\mathfrak{p}}$ from $\hat{\mathfrak{p}}$, by an argument similar to the proof of [19, Lemma 8.4], we may assume that the natural isomorphism

$$
H^{0}\left(D, f^{*} \mathcal{O}_{\mathbb{C P}^{4}}(1) \otimes W\right) \simeq H^{0}\left(D, f^{*} \mathcal{O}_{\mathbb{C P}^{4}}(1)\right) \otimes Y
$$

preserves orientation. Here, we have used the right-to-left lexicographical orientation of the tensor product. Up to a degree independent sign, we may assume that 
the isomorphism

$$
H^{0}(D, \mathcal{O}(1) \otimes U) \simeq H^{0}(D, \mathcal{O}(1)) \otimes X
$$

also preserves sign. So, it remains to compute the third term of the exact sequence,

$$
0 \rightarrow H^{0}(D, \mathcal{O}(1)) \otimes X \stackrel{d \tilde{f}}{\rightarrow} H^{0}(D, \mathcal{O}(p)) \otimes Y \rightarrow \operatorname{Def}(D, f) \rightarrow 0
$$

from the first two.

We assume without loss of generality that the induced $\mathbf{T}^{2}$-action on

$$
H^{0}\left(D, f^{*} \mathcal{O}_{\mathbb{C P}^{4}}(1)\right) \simeq H^{0}(D, \mathcal{O}(p))
$$

has weights

$$
\frac{\lambda_{1}}{p}, \frac{3 \lambda_{1}}{p}, \ldots,
$$

as opposed to their negatives. That is, we assume that the action on the underlying vector space considered in Section 4.2 has weight $\lambda=\lambda_{1} / p$. This depends on the choice of $\hat{\mathfrak{p}}$. One way or another, the opposite disk has the opposite sign, so we can always interchange $\zeta_{1}$ and $\zeta_{2}$ to satisfy our assumption. Note, however, that the full localization contribution of $[f]$ including the weights of the obstruction bundle is invariant under the symmetry

$$
\lambda_{1} \mapsto \lambda_{2}=-\lambda_{1}, \quad \lambda_{3} \mapsto \lambda_{4}=-\lambda_{3} .
$$

Let $Y^{\prime} \subset Y$ denote the 2-dimensional linear subspace corresponding to $V_{\lambda_{1}}$ and let $Y^{\prime \prime} \simeq V_{\lambda_{3}} \oplus \mathbb{R}$ denote its $\mathbf{T}^{2}$-invariant complement. Note that

$$
\operatorname{Im}(d \tilde{f}) \subset H^{0}(D, \mathcal{O}(p)) \otimes Y^{\prime} .
$$

We study the induced morphism,

$$
d \tilde{f}^{\prime}: H^{0}(D, \mathcal{O}(1)) \otimes X \rightarrow H^{0}(D, \mathcal{O}(p)) \otimes Y^{\prime}
$$

carefully in order to calculate the weights of the cokernel with attention to sign necessary because of the trivial representations that occur in the domain and in the range. Indeed, by formulas (17) and (14), we have

$$
\begin{gathered}
H^{0}(D, \mathcal{O}(1)) \otimes X \simeq V_{0} \oplus V_{2 \lambda_{1} / p} \\
H^{0}(D, \mathcal{O}(p)) \otimes Y^{\prime} \simeq \bigoplus_{i=0}^{(p-1) / 2} V_{(2 i+1+p) \lambda_{1} / p} \oplus \bigoplus_{i=0}^{(p-3) / 2} V_{(2 i+1-p) \lambda_{1} / p} \oplus V_{0} .
\end{gathered}
$$

The map $d \tilde{f}^{\prime}$ is determined up to homothety on the summand $V_{2 \lambda_{1} / p}$ by $\mathbf{T}^{2}$. equivariance. Since, homotheties of an even-dimensional vector space preserve orientation, we need calculate no further. However, we need more information to determine $d \tilde{f}^{\prime}$ on the trivial representation summand $V_{0}$. It is possible to explicitly write oriented bases of $X$ and $Y^{\prime}$ and see that $d \tilde{f}^{\prime}$ preserves orientation on the summand $V_{0}$. However, that would lead to considerable notational complications. Our strategy is to modify the action of $\mathbf{T}^{2}$ on $X$ and $Y^{\prime}$ so that $d \tilde{f}$ is still equivariant, but there are no copies of the trivial representation in the decomposition to irreducibles. To check equivariance, we may work over the complex numbers, thus simplifying formulas.

Choose coordinates $z_{1}$ and $z_{2}$ on $U$ such that under the action of $\mathbf{T}^{2}$,

$$
z_{1} \mapsto e^{i \lambda_{1} / p} z_{1}, \quad z_{2} \mapsto e^{-i \lambda_{1} / p} z_{2}
$$


Then $f$ is given explicitly by

$$
\left[z_{1}: z_{2}\right] \mapsto\left[z_{1}^{p}: z_{2}^{p}: 0: 0: 0\right] .
$$

Let $e_{1}, e_{2}$, be a basis of $U$, dual to $z_{1}, z_{2}$. A section

$$
\xi \in H^{0}\left(\mathbb{C P}^{1}, \mathcal{O}(1) \otimes U\right)
$$

takes the form

$$
\xi=\xi_{1} e_{1} \oplus \xi_{2} e_{2}
$$

where $\xi_{1}, \xi_{2}$, are linear functions on $U$. Let $c_{1}, c_{2}$, be the basis of $Y^{\prime}$ corresponding to $e_{1}, e_{2}$. Then

$$
d \tilde{f}^{\prime}(\xi)=\xi_{1} p z_{1}^{p-1} c_{1}+\xi_{2} p z_{2}^{p-1} c_{2} .
$$

In the following, we denote by $\epsilon$ a small rational number. If we allow $\mathbf{T}^{2}$ to act on $X$ by $\lambda_{1}(1 / p+\epsilon)$ and on $Y^{\prime}$ by $\lambda_{1}(1+\epsilon)$, then $d \tilde{f}$ will remain equivariant. The summands $V_{0}$ in decompositions (20) both change to $V_{-\lambda_{1} \epsilon}$. Choosing $\epsilon$ small enough, we may assume that no new trivial summands appear. Since the direct sum decomposition doesn't change on the level of vector spaces as we change weights, we conclude that $d \tilde{f}^{\prime}$ maps $V_{0}$ to $V_{0}$ preserving orientation. Hence, the cokernel of $d \tilde{f}^{\prime}$ has equivariant Euler class,

$$
\begin{aligned}
e\left(\operatorname{coker}\left(d \tilde{f}^{\prime}\right)\right)= & \lambda_{1}^{p-1} p^{1-p}(p+1)(p+3) \ldots(2 p) \\
& \times(1-p)(3-p) \ldots(-4) \\
= & (-1)^{(p-1) / 2} 2^{p-1} p ! p^{1-p} \lambda_{1}^{p-1} .
\end{aligned}
$$

Now, $H^{0}(D, \mathcal{O}(p)) \otimes Y^{\prime \prime}$ contributes directly to $\operatorname{Def}(D, f)$ as follows. Decomposing $Y^{\prime \prime} \simeq V_{\lambda_{3}} \oplus \mathbb{R}$, and using formulas (17) and (14), we calculate

$$
\begin{aligned}
e\left(H^{0}(D, \mathcal{O}(p)) \otimes V_{\lambda_{3}}\right)=\prod_{i=0}^{(p-1) / 2}( & \left.\left(1-\frac{2 i}{p}\right) \lambda_{1}-\lambda_{3}\right) \\
& \times\left(\left(1-\frac{2 i}{p}\right) \lambda_{1}+\lambda_{3}\right)
\end{aligned}
$$

and

$$
e\left(H^{0}(D, \mathcal{O}(p)) \otimes \mathbb{R}\right)=p ! ! p^{-(p+1) / 2} \lambda_{1}^{(p+1) / 2} .
$$

In conclusion,

$$
\begin{aligned}
& e\left(N_{f}\right)=(-1)^{(p-1) / 2} 2^{p-1} p^{-(3 p-1) / 2} p ! p ! ! \lambda_{1}^{(3 p-1) / 2} \\
& \times \prod_{i=0}^{(p-1) / 2}\left(\left(1-\frac{2 i}{p}\right) \lambda_{1}-\lambda_{3}\right)\left(\left(1-\frac{2 i}{p}\right) \lambda_{1}+\lambda_{3}\right) .
\end{aligned}
$$

On the other hand, by (14) the Euler class of the obstruction bundle is just

$$
e\left(\left(F_{d}\right)_{f}\right)=e\left(H^{0}(D, \mathcal{O}(5 p))\right)=(5 p) ! ! p^{-(5 p+1) / 2} \lambda_{1}^{(5 p+1) / 2} .
$$

Here, we are assuming that the Pin structure that $\mathfrak{p}^{\prime}$ induces on

$$
\mathcal{O}(5 p)_{\mathbb{R}} \simeq f^{*} \mathcal{O}_{\mathbb{C P}^{4}}(5)_{\mathbb{R}}
$$

agrees with $\mathfrak{p}_{-1}$. If for a given $\mathfrak{p}^{\prime}$ this is not true, reversing $\mathfrak{p}^{\prime}$ changes the sign of $e\left(\left(F_{d}\right)_{f}\right)$ by -1 for all $p$ by [19, Lemma 2.10]. So, we reverse $\mathfrak{s}$, thus reversing $\mathfrak{p}^{\prime}$. 
Hence, the dependence of the total sign of $I\left(\zeta_{1}, p\right)$ on $\mathfrak{s}$ is as claimed. Combining everything, we obtain

$$
I\left(\zeta_{1}, p\right)=4 \frac{(-1)^{\frac{p-1}{2}}}{p} \frac{\frac{(5 p) ! !}{p ! p ! !}\left(\frac{\lambda_{1}}{2 p}\right)^{p+1}}{\prod_{i=0}^{(p-1) / 2}\left(\left(1-\frac{2 i}{p}\right) \lambda_{1}-\lambda_{3}\right)\left(\left(1-\frac{2 i}{p}\right) \lambda_{1}+\lambda_{3}\right)} .
$$

The extra factor of $\frac{1}{p}$ comes from the orbifold structure of the moduli space $\widetilde{M}_{D}\left(\mathbb{C P}^{4} / \mathbb{C P}_{\mathbb{R}}^{4}, d\right)$ at $[f]$.

\section{Proof of the Euler Class formula}

5.1. Construction of $\widetilde{M}_{D}\left(\mathbb{C P}^{4} / \mathbb{C P}_{\mathbb{R}}^{4}, d\right)$. A detailed construction was not required in [19] to prove the invariance of $N_{d}^{d i s k}$. However, to apply the Atiyah-Bott localization formula as in the proof of Theorem 1 and Proposition [19, as well as for the obstruction bundle argument in the proof of Theorem 3, we need the following result.

Proposition 11. $\widetilde{M}_{D}\left(\mathbb{C P}^{4} / \mathbb{C P}_{\mathbb{R}}^{4}, d\right)$ is a smooth closed orbifold and $F_{d}$ is a smooth orbibundle.

Proof. First, we give a detailed definition of $\widetilde{M}_{D}\left(\mathbb{C P}^{4} / \mathbb{C} \mathbb{P}_{\mathbb{R}}^{4}, d\right)$. The moduli space $\bar{M}_{D}\left(\mathbb{C P}^{4} / \mathbb{C} \mathbb{P}_{\mathbb{R}}^{4}, d\right)$ is a smooth orbifold with corners. A point in a corner of codimension $k$ corresponds to an open stable map with $k+1$ disk components. Such a stable map may have arbitrarily many sphere components. However, since we consider open stable maps of genus 0 , each sphere component belongs to a tree of sphere components attached to a unique disk. We define the total degree of a disk component to be its own degree plus the degree of all attached spheres components.

We classify corners of $\bar{M}_{D}\left(\mathbb{C P}^{4} / \mathbb{C P}_{\mathbb{R}}^{4}, d\right)$ by the intersection type $I$ of the open stable maps. The intersection type $I=\left(T_{I}, \ell_{I}\right)$ of an open stable map $f$ of genus zero consists of a tree $T_{I}$ and a labelling $\ell_{I}$ of the vertices of $T_{I}$ by nonnegative integers. The vertices of the tree correspond to disk maps and the edges correspond to nodes connecting two disks. The labelling of a vertex is the total degree of the corresponding disk component. We denote by $|I|$ the number of vertices of $T_{I}$ the codimension of the corresponding corner. Let $I$ be an intersection type, and let $e$ be an edge of $T_{I}$ connecting vertices $v_{1}$ and $v_{2}$. Gluing $I$ at $e$ to obtain $I^{\prime}$ means contracting $e$ so that $v_{1}$ and $v_{2}$ become a single vertex $v$ of $I^{\prime}$ and defining $\ell_{I^{\prime}}(v)=\ell_{I}\left(v_{1}\right)+\ell_{I}\left(v_{2}\right)$. We define a partial ordering on the set of intersection types by $I_{1}<I_{2}$ if $I_{1}$ may be obtained from $I_{2}$ by a sequence of gluings. We use the notation $\partial_{I} M_{D}\left(\mathbb{C P}^{4} / \mathbb{C P}_{\mathbb{R}}^{4}, d\right)$ to denote the corner of $\bar{M}_{D}\left(\mathbb{C P}^{4} / \mathbb{C P}_{\mathbb{R}}^{4}, d\right)$ of intersection type $I$.

We refer to codimension one corners as boundary components. Points of the boundary correspond to stable maps with two disk components. The intersection type $I,|I|=2$, of such a stable map is essentially an unordered pair $\left\{d_{1}, d_{2}\right\}$, where the numbers $d_{i}$ are the total degrees of each disk component. Here $d_{1}+d_{2}=d$. For each possible such $I$, we define a smooth involution $c_{I}$ of the corresponding boundary component as follows. Choose a vertex $v_{I}$ of $T_{I}$. Since the total degree is odd, $v_{I}$ can be chosen as the unique vertex of odd degree. For

$$
[f] \in \partial_{I} M_{D}\left(\mathbb{C P}^{4} / \mathbb{C P}_{\mathbb{R}}^{4}, d\right),
$$


define $c_{I}(f)$ to be the open stable map obtained by replacing the disk component corresponding to $v_{I}$ and all attached sphere maps by their conjugates.

We extend $c_{I}$ to the closure

$$
\overline{\partial_{I} M_{D}\left(\mathbb{C P}^{4} / \mathbb{C P}_{\mathbb{R}}^{4}, d\right)}=\bigcup_{I^{\prime} \geq I} \partial_{I^{\prime}} M_{D}\left(\mathbb{C P}^{4} / \mathbb{C P}_{\mathbb{R}}^{4}, d\right)
$$

as follows. Let $[f] \in \partial_{I^{\prime}} M_{D}\left(\mathbb{C P}^{4} / \mathbb{C P}_{\mathbb{R}}^{4}, d\right)$ for some $I^{\prime}>I$. Let $\left\{v_{I^{\prime}}^{i}\right\}$ be the set of vertices of $I^{\prime}$ that glue to form $v_{I}$. We define $c_{I}(f)$ to be the open stable map obtained by replacing the disk-maps corresponding to the $v_{I^{\prime}}^{i}$ and all attached spheres maps by their conjugates.

Next, we define an equivalence relation $\sim$ on $\bar{M}_{D}\left(\mathbb{C P}^{4} / \mathbb{C P}_{\mathbb{R}}^{4}, d\right)$ as follows. Let $[p],[q] \in \partial_{I^{\prime}} M_{D}\left(\mathbb{C P}^{4} / \mathbb{C P}_{\mathbb{R}}^{4}, d\right)$. We write $p \sim q$ if there exists $I \leq I^{\prime}$ with $|I|=2$ such that $c_{I}(p)=q$. Finally, we can define

$$
\widetilde{M}_{D}\left(\mathbb{C P}^{4} / \mathbb{C P}_{\mathbb{R}}^{4}, d\right)=\bar{M}_{D}\left(\mathbb{C P}^{4} / \mathbb{C P}_{\mathbb{R}}^{4}, d\right) / \sim .
$$

We now prove $\widetilde{M}_{D}\left(\mathbb{C P}^{4} / \mathbb{C P}_{\mathbb{R}}^{4}, d\right)$ is a closed orbifold. We refer the reader to 4. Section 2] for a quick review of orbifolds. We essentially follow the notation established there. Let

$$
\pi: \bar{M}_{D}\left(\mathbb{C P}^{4} / \mathbb{C P}_{\mathbb{R}}^{4}, d\right) \rightarrow \widetilde{M}_{D}\left(\mathbb{C P}^{4} / \mathbb{C P}_{\mathbb{R}}^{4}, d\right)
$$

denote the quotient projection. Being a closed orbifold is a local property, so we restrict our attention to a small neighborhood of a point

$$
[p] \in \widetilde{M}_{D}\left(\mathbb{C P}^{4} / \mathbb{C P}_{\mathbb{R}}^{4}, d\right) .
$$

If $\pi^{-1}([p])$ lies away from the corners of $\bar{M}_{D}\left(\mathbb{C P}^{4} / \mathbb{C P}_{\mathbb{R}}^{4}, d\right)$, there is nothing to prove.

If $\pi^{-1}([p])$ meets the corners, we construct an orbifold chart in a neighborhood of $[p]$ in a canonical way. Let $[\tilde{p}] \in \pi^{-1}([p])$. We assume

$$
[\tilde{p}] \in \partial_{I_{p}} M_{D}\left(\mathbb{C P}^{4} / \mathbb{C P}_{\mathbb{R}}^{4}, d\right), \quad k=\left|I_{p}\right|-1 \geq 1 .
$$

Let $\left(V_{\tilde{p}}, \Gamma_{\tilde{p}}, \psi_{\tilde{p}}\right)$ be an orbifold chart on $\bar{M}_{D}\left(\mathbb{C P}^{4} / \mathbb{C P}_{\mathbb{R}}^{4}, d\right)$ at $\tilde{p}$. Here $V_{\tilde{p}}$ is a neighborhood of 0 in

$$
\mathbb{R}_{+k}^{n}=\left\{\left(x_{1}, \ldots, x_{n}\right) \in \mathbb{R}^{n} \mid x_{i} \geq 0, i=1, \ldots, k\right\},
$$

$\Gamma_{\tilde{p}}$ is a finite group acting on $V_{\tilde{p}}$, and $\psi_{\tilde{p}}$ is a $\Gamma_{\tilde{p}}$ invariant homomorphism from $V_{\tilde{p}}$ to a neighborhood of $[\tilde{p}]$. From the definition of the orbifold structure on $\bar{M}_{D}\left(\mathbb{C P}^{4} / \mathbb{C P}_{\mathbb{R}}^{4}, d\right)$, the groups $\Gamma_{\tilde{p}}$ for $[\tilde{p}] \in \pi^{-1}([p])$ are all isomorphic. So, we may define a group $\widetilde{\Gamma}_{p}$ with isomorphisms $\widetilde{\Gamma}_{p} \simeq \Gamma_{\tilde{p}}$. We define

$$
\partial_{I} V_{\tilde{p}}=\psi_{\tilde{p}}^{-1}\left(\partial_{I} M_{D}\left(\mathbb{C P}^{4} / \mathbb{C P}_{\mathbb{R}}^{4}, d\right)\right) .
$$

By definition of an orbifold with corners, $\partial_{I} V_{\tilde{p}}$ is contained in a subset of $\mathbb{R}_{+k}^{n}$ where $|I|-1$ of the coordinates $x_{1}, \ldots, x_{k}$, are zero. By definition of a smooth map of an orbifold, possibly shrinking the charts $V_{\tilde{p}}$, we may assume that $c_{I}$ induces a smooth $\widetilde{\Gamma}_{p}$ equivariant involution of the disjoint union of the corners $\partial_{I^{\prime}} V_{\tilde{p}}, I^{\prime} \geq I$, over all $[\tilde{p}] \in \pi^{-1}([p])$.

Let $I_{1}, \ldots, I_{k}$ enumerate the set of $I \leq I_{p}$ such that $|I|=2$, the intersection types of the boundary components adjacent to $p$. For a multi-index

$$
E=\left(\epsilon_{1}, \ldots, \epsilon_{k}\right) \in(\mathbb{Z} / 2 \mathbb{Z})^{k},
$$


we define an involution $c_{E}$ of $\partial_{I_{p}} M_{D}\left(\mathbb{C P}^{4} / \mathbb{C P}_{\mathbb{R}}^{4}, d\right)$ by

$$
c_{E}=\prod_{j=1}^{k} c_{I_{j}}^{\epsilon_{j}}
$$

So, the group $(\mathbb{Z} / 2 \mathbb{Z})^{k}$ acts on $\partial_{I_{p}} M_{D}\left(\mathbb{C P}^{4} / \mathbb{C P}_{\mathbb{R}}^{4}, d\right)$ and, by definition of $\pi$, acts transitively on $\pi^{-1}([p])$. Define

$$
G_{p} \subset(\mathbb{Z} / 2 \mathbb{Z})^{k}
$$

to be the stationary subgroup of $[\tilde{p}] \in \pi^{-1}([p])$. The definition does not depend on the choice of $[\tilde{p}]$ because we are considering a transitive action of an abelian group. Define

$$
\hat{V}_{p}=\coprod_{[\tilde{p}] \in \pi^{-1}([p])} V_{\tilde{p}} \times G_{p}, \quad V_{p}=\hat{V}_{p} / \sim^{*},
$$

where the equivalence relation $\sim^{*}$ is defined as follows. Let

$$
E_{j} \in(\mathbb{Z} / 2 \mathbb{Z})^{k}
$$

denote the multi-index with $\epsilon_{i}=0$ for $i \neq j$ and $\epsilon_{j}=1$. For

$$
(q, E),\left(q^{\prime}, E^{\prime}\right) \in \hat{V}_{p}
$$

define $(q, E) \sim^{*}\left(q^{\prime}, E^{\prime}\right)$ if $q=c_{I_{j}}\left(q^{\prime}\right)$ and $E=E^{\prime}+E_{j}$ for some $j$. Define

$$
\Gamma_{p}=\widetilde{\Gamma}_{p} \times G_{p} .
$$

The group $\Gamma_{p}$ acts naturally on $V_{p}$. Finally, let $\psi_{p}$ be the $\Gamma_{p}$ invariant map from $V_{p}$ to $\widetilde{M}_{D}\left(\mathbb{C P}^{4} / \mathbb{C P}_{\mathbb{R}}^{4}, d\right)$ naturally induced by the $\psi_{\tilde{p}}$. Since $V_{p}$ is obtained by gluing together $2^{k}$ neighborhoods of $0 \in \mathbb{R}_{+k}^{n}$ on matching corners, $V_{p}$ is a neighborhood of $0 \in \mathbb{R}^{n}$. The triple $\left(V_{p}, \Gamma_{p}, \psi_{p}\right)$ is easily seen to specify a natural orbifold structure in a neighborhood of $p$.

The involutions $c_{I}$ lift naturally to the bundles

$$
\left.\hat{F}_{d}\right|_{\partial_{I} M_{D}\left(\mathbb{C P} \mathbb{P}^{4} / \mathbb{C P}_{\mathbb{R}}^{4}, d\right)} .
$$

Then, the exact same proof extends to construct the structure of an orbibundle on $F_{d}$.

In the following, $\mathfrak{p}$ denotes a Pin structure on $\mathbb{C P}_{\mathbb{R}}^{4}, \mathfrak{p}^{\prime}$ denotes a Pin structure on $\mathcal{O}_{\mathbb{C P}^{4}}(5)_{\mathbb{R}}$ and $\mathfrak{s}$ denotes a Spin structure on $Q_{\mathbb{R}}$.

Lemma 12. Any two of $\mathfrak{s}, \mathfrak{p}, \mathfrak{p}^{\prime}$ determines the third.

Proof. By the adjunction formula, the normal bundle $N_{Q}$ of $Q$ in $\mathbb{C P}^{4}$ satisfies $\left.N_{Q} \simeq \mathcal{O}(5)\right|_{Q}$. So, the lemma follows from the exact sequence,

$$
0 \rightarrow T Q_{\mathbb{R}} \rightarrow T \mathbb{C P}_{\mathbb{R}}^{4} \rightarrow N_{Q_{\mathbb{R}}} \rightarrow 0,
$$

and [19, Lemma 8.1].

We denote the determinant of the tangent bundle of $\widetilde{M}_{D}\left(\mathbb{C P}^{4} / \mathbb{C P}_{\mathbb{R}}^{4}, d\right)$ by $\mathcal{L}$.

Lemma 13. There exists a topological isomorphism $\operatorname{det} F_{d} \simeq \mathcal{L}$. Moreover, such an isomorphism is determined canonically up to homotopy by the choice of a Spin structure on $Q_{\mathbb{R}}$. 
Proof. Choose $\mathfrak{p}$ and $\mathfrak{p}^{\prime}$ compatible with $\mathfrak{s}$. Then $\mathfrak{p}$ induces an orientation on $\bar{M}_{D}\left(\mathbb{C P}^{4} / \mathbb{C P}_{\mathbb{R}}^{4}, d\right)$ and $\mathfrak{p}^{\prime}$ induces an orientation on $\hat{F}_{d}$. So, there exists a unique up to homotopy isomorphism,

$$
\operatorname{det}\left(\bar{M}_{D}\left(\mathbb{C P}^{4} / \mathbb{C P}_{\mathbb{R}}^{4}, d\right)\right) \simeq \operatorname{det}\left(\hat{F}_{d}\right),
$$

preserving orientation. We must check that the identifications involved in the construction of $\widetilde{M}_{D}\left(\mathbb{C P}^{4} / \mathbb{C P}_{\mathbb{R}}^{4}, d\right)$ are compatible with the above isomorphism: the sign of $c_{I}$ on $\partial_{I} M_{D}\left(\mathbb{C P}^{4} / \mathbb{C P}_{\mathbb{R}}^{4}, d\right)$ must be the same as the sign of $c_{I}$ on $\left.\hat{F}_{d}\right|_{\partial_{I} M_{D}\left(\mathbb{C P}^{4} / \mathbb{C P}_{\mathbb{R}}^{4}, d\right)}$. This follows easily from [19, Lemma 2.12].

5.2. Kuranishi structures. 1 We denote by $\bar{M}_{D}\left(Q / Q_{\mathbb{R}}, d\right)$ the moduli space of open stable maps to $\left(Q, Q_{\mathbb{R}}\right)$. In general, the space $\bar{M}_{D}\left(Q / Q_{\mathbb{R}}, d\right)$ is a compact metrizable space. In the following proof, we use the theory of Kuranishi structures with corners developed in 4, 3, to define intersection theory on $\bar{M}_{D}\left(Q / Q_{\mathbb{R}}, d\right)$. The Kuranishi structures used here were shown to exist in [4, 3]. See 19, Appendix A and Section 7] for a very brief summary of this theory, from which we take our notational conventions. In the following, unless explicitly noted, all Kuranishi structures are Kuranishi structures with corners.

We will need the following definition, which is similar to the notion of an involution of a Kuranishi structure [19, Definition 7.1], but without property (E1). Suppose $(X, \mathcal{K})$ and $\left(X^{\prime}, \mathcal{K}^{\prime}\right)$ are spaces with Kuranishi structure

$$
\begin{aligned}
\mathcal{K} & =\left(V_{p}, E_{p}, \Gamma_{p}, s_{p}, \psi_{p}, V_{p q}, h_{p q}, \varphi_{p q}, \hat{\varphi}_{p q}\right), \\
\mathcal{K}^{\prime} & =\left(V_{p}^{\prime}, E_{p}^{\prime}, \Gamma_{p}^{\prime}, s_{p}^{\prime}, \psi_{p}^{\prime}, V_{p q}^{\prime}, h_{p q}^{\prime}, \varphi_{p q}^{\prime}, \hat{\varphi}_{p q}^{\prime}\right) .
\end{aligned}
$$

Let $f: X \rightarrow X^{\prime}$ be a continuous map.

Definition 14. An extension $\tilde{f}$ of $f$ to a map of spaces with Kuranishi structure consists of $\Gamma_{p}$-equivariant maps

$$
f_{p}: V_{p} \rightarrow V_{f(p)}^{\prime}, \quad \hat{f}_{p}: E_{p} \rightarrow E_{f(p)}^{\prime}
$$

covering $f_{p}$ such that

(M1) $s_{f(p)}^{\prime} \circ f_{p}=\hat{f}_{p} \circ s_{p}$.

(M2) $\left.\psi_{f(p)}^{\prime} \circ f_{p}\right|_{s_{p}^{-1}(0)}=f \circ \psi_{p}$.

(M3) $f_{q}$ maps $V_{p q} \subset V_{q}$ to $V_{f(p) f(q)}^{\prime} \subset V_{f(q)}^{\prime}$

(M4) $f_{p} \circ \varphi_{p q}=\varphi_{f(p) f(q)}^{\prime} \circ f_{q}$ and $\hat{f}_{p} \circ \hat{\varphi}_{p q}=\hat{\varphi}_{f(p) f(q)}^{\prime} \circ \hat{f}_{q}^{\prime}$.

Now, suppose that $(X, \mathcal{K})$ has a tangent bundle given by $\Phi_{p q}$ and $\left(X^{\prime}, \mathcal{K}^{\prime}\right)$ has a tangent bundle given by $\Phi_{p q}^{\prime}$. We say that $\tilde{f}$ is smooth if

$$
\Phi_{f(p) f(q)}^{\prime} \circ \hat{f}_{q}=\hat{f}_{p} \circ \Phi_{p q} .
$$

We say that $\tilde{f}$ is an embedding if $f_{p}$ are all embeddings and $\hat{f}_{p}$ are all injective bundle maps. The preceding definition of maps of spaces with Kuranishi structure is very rigid and not likely to make a very nice category. A better definition for a

\footnotetext{
${ }^{1}$ Here we use the virtual moduli cycle construction of [3], based on Kuranishi structures. In Sections 1.5 .3 we assume the coordinate transforms and Kuranishi maps are smooth, as explained in [3 Appendix A1.4]. Alternatively, we could assume only stratified smoothness, as explained in 20. We anticipate that with the completion of the generalized Fredholm theory currently being introduced in 8, 9, the ideas of Sections 15.3 will translate into a proof of Theorem 3 based on that framework.
} 
general morphism of spaces with Kuranishi structure would be something like the diagram of Figure 2,

We will also need the notion of vector bundles over a space with Kuranishi structure and their Euler classes. Vector bundles over a space with Kuranishi structure were constructed in a very general form in [4, Section 5] so as to include the tangent bundle to a Kuranishi structure. The bundles we use here correspond to the special case in which $F_{2}$ of 4 is taken to have rank 0 everywhere. We let $(X, \mathcal{K})$ denote a general space with Kuranishi structure as above.

Definition 15. A vector bundle $F$ over $(X, \mathcal{K})$ consists of

(1) For each $p \in X$, a $\Gamma_{p}$-equivariant vector bundle $F^{p} \rightarrow V_{p}$.

(2) For each $p \in X$ and $q \in \operatorname{Im} \psi_{p}$, an $h_{p q}$-equivariant vector bundle isomor$\operatorname{phism} \Phi_{p q}^{F}:\left.\left.F_{q}\right|_{V_{p q}} \rightarrow F_{p}\right|_{\operatorname{Im}\left(\varphi_{p q}\right)}$ covering $\varphi_{p q}$.

The Euler class of a vector bundle over a space with Kuranishi structure should determine a cohomology class in a cohomology theory for Kuranishi spaces. However, such a theory has not been developed. Since the Euler classes considered here always have critical dimension, essentially all the information in the Euler class is contained in a single number: the integral of the Euler class over the fundamental class. So, we focus on defining the integral of the Euler class.

Let $F$ denote a vector bundle over $(X, \mathcal{K})$ of rank equal to the expected dimension of $(X, \mathcal{K})$. Let $\mathcal{L}_{\mathcal{K}}$ denote the determinant of the tangent bundle of $(X, \mathcal{K})$, i.e., the line bundle over $(X, \mathcal{K})$ determined locally by the line bundles

$$
\operatorname{det}\left(T V_{p}\right) \otimes \operatorname{det}\left(E_{p}\right)^{*} \rightarrow V_{p}
$$

Assume that an isomorphism

$$
\operatorname{det}\left(\mathcal{L}_{\mathcal{K}}\right) \simeq \operatorname{det}(F)
$$

has been specified. Choose a transverse perturbation of the space with Kuranishi structure $(X, \mathcal{K})$. See [19, Theorem A.4] for a brief review of notation. Choose multivalued sections $\xi_{p}^{\prime}$ of $F_{p}^{\prime}$ such that the multi-valued section $\xi_{p}^{\prime}+s_{p, n}^{\prime}$ of $E_{p}^{\prime} \oplus F_{p}^{\prime}$ is transverse. Let $\sigma$ denote the 0-dimensional rational simplicial complex determined by the vanishing set of $\xi_{p}^{\prime}+s_{p, n}^{\prime}$. The orientation of $\sigma$ is determined by the isomorphism (21). Let $|\sigma|$ denote the rational weighted cardinality of $\sigma$.

Definition 16. We define the Euler class of $F$ by

$$
\int_{[X, \mathcal{K}]} e(F)=|\sigma| \text {. }
$$

Straightforward cobordism arguments show the definition does not depend on the choice of section $\xi$ or the perturbation of Kuranishi structure. See 4, Sections 4 and 17].

5.3. Proof of Theorem 3. We continue to employ the notation of the proof of Proposition [1]. Since $\bar{M}_{D}\left(Q / Q_{\mathbb{R}}, d\right)$ consists of open stable maps, we may define

$$
\partial_{I} M_{D}\left(Q / Q_{\mathbb{R}}, d\right) \subset \bar{M}_{D}\left(Q / Q_{\mathbb{R}}, d\right)
$$

to be the subspace consisting of all open stable maps of intersection type $I$. Similarly, we may define the involution $c_{I}$ of the corner of intersection type $I^{\prime}$ for $I^{\prime} \geq I$, and the quotient space $\widetilde{M}_{D}\left(Q / Q_{\mathbb{R}}, d\right)$. Let $\mathcal{K}_{Q}$ be a Kuranishi structure with corners on $\bar{M}_{D}\left(Q / Q_{\mathbb{R}}, d\right)$. By the arguments of [19, Section 7], the involutions 


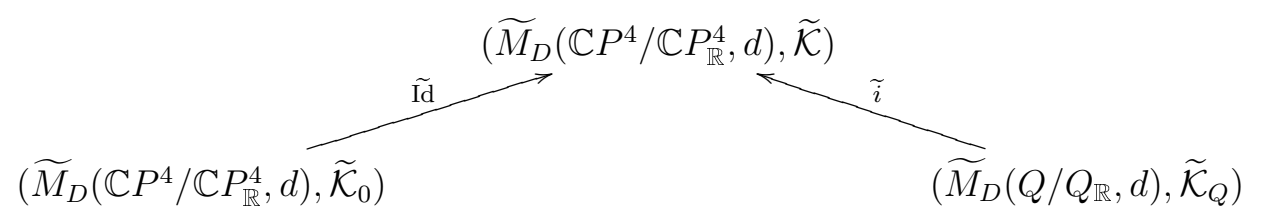

Figure 2

$c_{I}$ extend smoothly to the Kuranishi structure $\left.\mathcal{K}_{Q}\right|_{\overline{\partial_{I} M_{D}\left(Q / Q_{\mathbb{R}}, d\right)}}$. Recapitulating the proof of Proposition 11, $\mathcal{K}_{Q}$ induces a Kuranishi structure without boundary $\widetilde{\mathcal{K}}_{Q}$ on $\widetilde{M}_{D}\left(Q / Q_{\mathbb{R}}, d\right)$. A transverse perturbation of the space with Kuranishi structure, $\left(\widetilde{M}_{D}\left(Q / Q_{\mathbb{R}}, d\right), \widetilde{\mathcal{K}}_{Q}\right)$, defines a simplicial complex consisting of a finite number of 0 -simplices with rational weights. The weighted count of the 0 -simplices is $N_{d}^{\text {disk }}$. While the definition is not exactly the same as the definition given in [19, the equivalence is not hard to verify.

An orbifold structure is a special case of a Kuranishi structure for which all the bundles $E_{p}$ are rank 0 . Let $\mathcal{K}_{0}$ denote the Kuranishi structure on $\bar{M}_{D}\left(\mathbb{C P}^{4} / \mathbb{C P}_{\mathbb{R}}^{4}, d\right)$ coming from the orbifold structure, and let $\widetilde{\mathcal{K}}_{0}$ denote the Kuranishi structure on $\widetilde{M}_{D}\left(\mathbb{C P}^{4} / \mathbb{C P}_{\mathbb{R}}^{4}, d\right)$ coming from the orbifold structure constructed in Proposition 11 . Also, let

$$
i: \widetilde{M}_{D}\left(Q / Q_{\mathbb{R}}, d\right) \rightarrow \widetilde{M}_{D}\left(\mathbb{C P}^{4} / \mathbb{C P}_{\mathbb{R}}^{4}, d\right)
$$

denote the natural inclusion. We would like to construct a Kuranishi structure $\widetilde{\mathcal{K}}$ on $\widetilde{M}_{D}\left(\mathbb{C P}^{4} / \mathbb{C P}_{\mathbb{R}}^{4}, d\right)$ for which we have the diagram of spaces with Kuranishi structure shown in Figure 2 .

The structure $\widetilde{\mathcal{K}}$ may be obtained from a Kuranishi structure on $\bar{M}_{D}\left(\mathbb{C P}^{4} / \mathbb{C P}_{\mathbb{R}}^{4}, d\right)$ that admits extensions of the involutions $c_{I}$ for which the diagram of Figure 2 holds with tildes replaced by bars. For each $[p] \in \bar{M}_{D}\left(Q / Q_{\mathbb{R}}, d\right)$, we extend the Kuranishi neighborhood

$$
\left(V_{p}^{Q}, E_{p}^{Q}, \Gamma_{p}^{Q}, s_{p}^{Q}, \psi_{p}^{Q}\right)
$$

given by $\mathcal{K}_{Q}$ to a Kuranishi neighborhood $\left(V_{p}, E_{p}, \Gamma_{p}, s_{p}, \psi_{p}\right)$ for the space $\bar{M}_{D}\left(\mathbb{C P}^{4} / \mathbb{C P}_{\mathbb{R}}^{4}, d\right)$. We detail the construction of the extension for $p$ an irreducible stable map. The construction for $p$ a reducible stable map is similar but notationally more complicated.

Let $B_{D}\left(Q / Q_{\mathbb{R}}, d\right)$ and $B_{D}\left(\mathbb{C P}^{4} / \mathbb{C P}_{\mathbb{R}}^{4}, d\right)$ denote the Banach manifolds of $W^{1, r}$ maps

$$
(D, \partial D) \rightarrow\left(Q, Q_{\mathbb{R}}\right), \quad(D, \partial D) \rightarrow\left(\mathbb{C P}^{4}, \mathbb{C P}_{\mathbb{R}}^{4}\right),
$$

respectively. Let

$$
\mathcal{E}^{Q} \rightarrow B_{D}\left(Q / Q_{\mathbb{R}}, d\right), \quad \mathcal{E} \rightarrow B_{D}\left(\mathbb{C P}^{4} / \mathbb{C P}_{\mathbb{R}}^{4}, d\right),
$$

be defined fiberwise by

$$
\begin{aligned}
\mathcal{E}_{f}^{Q} & =L^{r}\left(D, \Omega^{0,1}\left(f^{*} T Q\right)\right), \quad f \in B_{D}\left(Q / Q_{\mathbb{R}}, d\right), \\
\mathcal{E}_{f} & =L^{r}\left(D, \Omega^{0,1}\left(f^{*} T \mathbb{C} P^{4}\right)\right), \quad f \in B_{D}\left(\mathbb{C P}^{4} / \mathbb{C P}_{\mathbb{R}}^{4}, d\right) .
\end{aligned}
$$

Let $\bar{\partial}^{Q}$ (resp. $\bar{\partial}$ ) denote the section of $\mathcal{E}^{Q}$ (resp. $\mathcal{E}$ ) given by the nonlinear CauchyRiemann operator on maps to $Q$ (resp. $\mathbb{C P}^{4}$ ). Let $D \bar{\partial}^{Q}$ and $D \bar{\partial}$ denote choices of the vertical parts of their respective linearizations. 
We briefly outline the construction [4, 3] of the Kuranishi neighborhood $\left(V_{p}^{Q}, E_{p}^{Q}\right.$, $\left.\Gamma_{p}^{Q}, s_{p}^{Q}, \psi_{p}^{Q}\right)$ in order to explain how to extend it. First, choose a finite-dimensional subspace $\hat{E}_{p}^{Q} \subset \mathcal{E}_{p}^{Q}$ so that

$$
D \bar{\partial}^{Q}: T_{p} B_{D}\left(Q / Q_{\mathbb{R}}, d\right) \rightarrow \mathcal{E}_{p}^{Q} / \hat{E}_{p}^{Q}
$$

is surjective. Extend $\hat{E}_{p}^{Q}$ to a vector bundle $\check{E}_{p}^{Q}$ over a neighborhood of $p$ in $B_{D}\left(Q / Q_{\mathbb{R}}, d\right)$ by parallel transport. Over a sufficiently small neighborhood of $p$, $D \bar{\partial}^{Q}$ surjects onto $\mathcal{E}^{Q} / \check{E}_{p}^{Q}$. So, we may define a smooth manifold

$$
\widetilde{V}_{p}^{Q}=\left(\bar{\partial}^{Q}\right)^{-1}\left(\check{E}_{p}^{Q}\right) .
$$

We define $V_{p}^{Q}$ to be an appropriate section of the action of the infinitesimal reparametrization group on $\widetilde{V}_{p}^{Q}$. Then, we take $E_{p}^{Q}=\left.\check{E}_{p}^{Q}\right|_{V_{p}^{Q}}$ and

$$
s_{p}^{Q}(x)=\bar{\partial}^{Q}(x) \in E_{p}^{Q}, \quad x \in V_{p}^{Q} .
$$

The group $\Gamma_{p}^{Q}$ arises from the remaining discrete part of the reparametrization group of $p$.

To extend the Kuranishi neighborhood $\left(V_{p}^{Q}, E_{p}^{Q}, \Gamma_{p}^{Q}, s_{p}^{Q}, \psi_{p}^{Q}\right)$ to a Kuranishi neighborhood for $\bar{M}_{D}\left(\mathbb{C P}^{4} / \mathbb{C P}_{\mathbb{R}}^{4}, d\right)$, we extend $\check{E}_{p}^{Q}$ by parallel translation to a vector bundle $\check{E}_{p}$ over a neighborhood of $p$ in $B_{D}\left(\mathbb{C P}^{4} / \mathbb{C P}_{\mathbb{R}}^{4}, d\right)$. Since $\mathbb{C P}^{4}$ is a homogeneous space, the operator $D \bar{\partial}$ is surjective onto $\mathcal{E}_{p}$ at every $[p] \in M_{D}\left(\mathbb{C P}^{4} / \mathbb{C P}_{\mathbb{R}}^{4}, d\right)$. So, we may define a smooth manifold

$$
\widetilde{V}_{p}=(\bar{\partial})^{-1}\left(\check{E}_{p}\right) \text {. }
$$

The definitions of $V_{p}, E_{p}, s_{p}$ and $\Gamma_{p}$ are just as before. In the case when $p$ is a reducible stable map, the extension procedure is similar except for one extra detail: we must be careful to perform parallel translations using a $c$-invariant metric so that the involutions $c_{I}$ extend to the corners of the extended Kuranishi neighborhood. See [19, Section 7] for a discussion of this issue.

For each point in $[p] \in \bar{M}_{D}\left(Q / Q_{\mathbb{R}}, d\right)$ we have just constructed a Kuranishi neighborhood $\left(V_{p}, E_{p}, \Gamma_{p}, s_{p}, \psi_{p}\right)$ for $\bar{M}_{D}\left(\mathbb{C P}^{4} / \mathbb{C P}_{\mathbb{R}}^{4}, d\right)$. It is straightforward to extend transition data $V_{p q}, h_{p q}, \phi_{p q}, \hat{\phi}_{p q}$ to these extended neighborhoods. In order to complete the construction of $\mathcal{K}$ it remains to define Kuranishi neighborhoods of points

$$
[p] \in \bar{M}_{D}\left(\mathbb{C P}^{4} / \mathbb{C P}_{\mathbb{R}}^{4}, d\right) \backslash \bar{M}_{D}\left(Q / Q_{\mathbb{R}}, d\right) .
$$

For such points, we take an orbifold coordinate chart as a Kuranishi neighborhood, letting $E_{p}$ be a trivial bundle of rank zero. Because the bundles $E_{p}$ are rank zero, it is again easy to construct the associated transition data $V_{p q}, h_{p q}, \phi_{p q}, \hat{\phi}_{p q}$. The existence of embeddings as in the diagram of Figure 2 follows immediately from the construction of $\mathcal{K}$.

We proceed to extend the bundle $F_{d}$ of Section 1.4 to a bundle $\widetilde{F}_{d}$ over the space with Kuranishi structure $\left(\widetilde{M}_{D}\left(\mathbb{C P}^{4} / \mathbb{C P}_{\mathbb{R}}^{4}, d\right), \widetilde{\mathcal{K}}\right)$. Again, it suffices to construct a bundle $\check{F}_{d}$ over the space with Kuranishi structure $\left(\bar{M}_{D}\left(\mathbb{C} P^{4} / \mathbb{C} P_{\mathbb{R}}^{4}, d\right), \mathcal{K}\right)$ so that $\check{F}_{d}$ admits an extension of the involutions $c_{I}$. We define the fiber of $\check{F}_{d}^{p}$ at $f \in V_{p}$ as follows. By construction, $f$ is a $W^{1, p}$-stable map satisfying the equation $\bar{\partial} f \in \widetilde{E}_{p}$. Since $f$ may not be holomorphic, we have to be careful how we define the complex structure on $f^{*} \mathcal{O}_{\mathbb{C} P^{4}}(5)$. Choose a $c$-invariant metric on $\mathcal{O}_{\mathbb{C} P^{4}}(5)$. The associated complex connection on $\mathcal{O}_{\mathbb{C} P^{4}}(5)$ will also be $c$-invariant. Equip $f^{*} \mathcal{O}_{\mathbb{C} P^{4}}(5)$ with the 
complex structure induced from the $(0,1)$ part of the pull-back connection. Then, we define as before,

$$
\left.\check{F}_{d}\right|_{f}=H^{0}\left(D, f^{*} \mathcal{O}_{\mathbb{C} P^{4}}(5)\right) .
$$

The transition functions $\Phi_{p q}^{F_{d}}$ are tautological. Because we used a $c$-invariant connection to induce the complex structure on $f^{*} \mathcal{O}_{\mathbb{C} P^{4}}(5)$, the involutions $c_{I}$ lift to involutions of $\left.\check{F}_{d}\right|_{\partial_{I^{\prime}} \bar{M}_{D}\left(\mathbb{C} P^{4} / \mathbb{C} P_{\mathbb{R}}^{4}, d\right)}$ for $I^{\prime} \geq I$. Hence, $\check{F}_{d}$ descends to a bundle $\widetilde{F}_{d}$ over $\left(\widetilde{M}_{D}\left(\mathbb{C} P^{4} / \mathbb{C} P_{\mathbb{R}}^{4}, d\right), \widetilde{\mathcal{K}}\right)$. Furthermore, the proof of Lemma 13 shows that the isomorphism $\operatorname{det}\left(F_{d}\right) \simeq \mathcal{L}$ extends to an isomorphism $\operatorname{det}\left(\widetilde{F}_{d}\right) \simeq \mathcal{L}_{\widetilde{\mathcal{K}}}$.

Let $\xi \in H^{0}\left(\mathbb{C P}^{4}, \mathcal{O}_{\mathbb{C P}^{4}}(5)\right)$ be the section defining the hypersurface $Q \subset \mathbb{C P}^{4}$. For general $f \in V_{p}$, the pull-back $f^{-1} \xi$ is not a holomorphic section of $f^{*} \mathcal{O}_{\mathbb{C P}}(5)$. Let

$$
P_{f}: W^{1, r}\left(D, f^{*} \mathcal{O}_{\mathbb{C P}^{4}}(5)\right) \rightarrow H^{0}\left(D, f^{*} \mathcal{O}_{\mathbb{C P}^{4}}(5)\right)
$$

denote the $L^{2}$ projection with respect to a $c$-invariant metric. We define a section $\check{\xi}_{p}$ of $\check{F}_{d}^{p}$ by

$$
\check{\xi}_{p}(f)=P_{f}\left(f^{-1} \xi\right), \quad f \in V_{p} .
$$

The local sections $\check{\xi}_{p}$ clearly match under the transition functions $\Phi_{p q}^{F_{d}}$ to define a global section $\check{\xi}$ of $\check{F}_{d}$. Since $\xi$ is $c$-invariant and $P_{f}$ is defined with respect to a $c$-invariant metric, we conclude that $\check{\xi}$ is compatible with the involutions $c_{I}$. So, $\check{\xi}$ descends to a section $\tilde{\xi}$ of $\widetilde{F}_{d}$.

Lemma 17. If the Kuranishi neighborhoods $V_{p}$ are chosen sufficiently small, then the sections $\check{\xi}_{p}$ vanish precisely on the image of the embedding of spaces with Kuranishi structure

$$
\left(\bar{M}_{D}\left(Q / Q_{\mathbb{R}}, d\right), \mathcal{K}^{Q}\right) \stackrel{\tilde{i}}{\rightarrow}\left(\bar{M}_{D}\left(\mathbb{C P}^{4} / \mathbb{C P}_{\mathbb{R}}^{4}, d\right), \mathcal{K}\right)
$$

Moreover, the sections $\check{\xi}_{p}$ are transverse to zero.

We postpone the proof of Lemma 17 until we complete the proof of Theorem 3 . Indeed, we calculate

$$
\int_{\left[\widetilde{M}_{D}\left(\mathbb{C P}^{4} / \mathbb{C P}_{\mathbb{R}}^{4}, d\right), \widetilde{\mathcal{K}}\right]} e\left(\widetilde{F}_{d}\right)
$$

in two different ways. On the one hand, we construct a transverse perturbation $\mathfrak{P}_{Q}$ of $\left(\widetilde{M}_{D}\left(Q / Q_{\mathbb{R}}, d\right), \widetilde{\mathcal{K}}^{Q}\right)$. Let $\sigma_{Q}$ denote the 0-dimensional simplicial complex defined by $\mathfrak{P}_{Q}$. By definition $N_{d}^{\text {disk }}=\left|\sigma_{Q}\right|$. Now, extend $\mathfrak{P}_{Q}$ to a transverse perturbation $\mathfrak{P}$ of $\left(\widetilde{M}_{D}\left(\mathbb{C P}^{4} / \mathbb{C P}_{\mathbb{R}}^{4}, d\right), \widetilde{\mathcal{K}}\right)$. By Lemma 17 , the local sections $\widetilde{\xi}_{p}^{\prime}+\tilde{s}_{p, n}^{\prime}$ of $\widetilde{F}_{d}^{p}+\widetilde{E}_{p}$ vanish transversely exactly on $\sigma_{Q}$. Assuming the following lemma, this implies that

$$
\int_{\left[\widetilde{M}_{D}\left(\mathbb{C P}^{4} / \mathbb{C P}_{\mathbb{R}}^{4}, d\right), \widetilde{\mathcal{K}}\right]} e\left(\widetilde{F}_{d}\right)=N_{d}^{d i s k} .
$$

Lemma 18. The orientation induced on each zero simplex in $\sigma_{Q}$ by the Kuranishi structure $\widetilde{\mathcal{K}}^{Q}$ agrees with the orientation induced by the Kuranishi structure $\widetilde{\mathcal{K}}$ and the vector bundle $\widetilde{F}_{d}$.

We postpone the proof of Lemma 18 until the end of the section.

On the other hand, the sections $\widetilde{s}_{p}$ of $\widetilde{E}_{p}$ are transverse without any perturbation since $\mathbb{C P}^{4}$ is convex. So, we may choose a transverse multi-valued section $\eta_{0}$ of $F_{d}$, 
and extend it to a multi-valued section $\tilde{\eta}$ of $\widetilde{F}_{d}$ over $\left(\widetilde{M}_{D}\left(\mathbb{C P}^{4} / \mathbb{C P}_{\mathbb{R}}^{4}, d\right), \widetilde{\mathcal{K}}\right)$ such that the local sections $\tilde{\eta}_{p}+\widetilde{s}_{p}$ will be transverse. This shows that

$$
\int_{\left[\widetilde{M}_{D}\left(\mathbb{C P}^{4} / \mathbb{C P}_{\mathbb{R}}^{4}, d\right), \widetilde{\mathcal{K}}\right]} e\left(\widetilde{F}_{d}\right)=\int_{\widetilde{M}_{D}\left(\mathbb{C} P^{4} / \mathbb{C} P_{\mathbb{R}}^{4}, d\right)} e\left(F_{d}\right) .
$$

Combining equations (23) and (24), we deduce Theorem 3 .

Proof of Lemma 17. We may focus on $\check{\xi}_{p}$ for $[p] \in \bar{M}_{D}\left(Q / Q_{\mathbb{R}}, d\right)$. Indeed, for other $p$, the Kuranishi neighborhood $V_{p}$ is just an orbifold chart on $\bar{M}_{D}\left(\mathbb{C P}^{4} / \mathbb{C P}_{\mathbb{R}}^{4}, d\right)$. So, for all $f \in V_{p}$ the pull-back $f^{-1} \xi$ is holomorphic and nonzero. So, $\check{\xi}_{p}(f)$ is never zero. In the case

$$
[p] \in \bar{M}_{D}\left(Q / Q_{\mathbb{R}}, d\right),
$$

we need to show that $\check{\xi}_{p}$ vanishes transversely on

$$
V_{p}^{Q} \subset V_{p}
$$

but nowhere else.

First, we establish some notation. Let $\nabla$ denote a complex $c$-invariant connection on $\mathcal{O}_{\mathbb{C P}^{4}}(5)$ and let $\nabla^{f}$ denote its pull-back to $f^{*} \mathcal{O}_{\mathbb{C P}^{4}}(5)$. Let $Y^{p} \rightarrow V_{p}$ and $Z^{p} \rightarrow V_{p}$ denote the Banach space bundles with fibers

$$
Y_{f}^{p}=W^{1, r}\left(D, f^{*} \mathcal{O}_{\mathbb{C P}^{4}}(5)\right), \quad Z_{f}^{p}=L^{r}\left(D, \Omega^{0,1}\left(f^{*} \mathcal{O}_{\mathbb{C P}^{4}}(5)\right)\right) .
$$

Define a map of Banach-space bundles

$$
d^{\prime \prime}: Y^{p} \rightarrow Z^{p}
$$

by

$$
d_{f}^{\prime \prime}=\left(\nabla^{f}\right)^{0,1}: W^{1, r}\left(D, f^{*} \mathcal{O}_{\mathbb{C P}^{4}}(5)\right) \rightarrow L^{r}\left(D, \Omega^{0,1}\left(f^{*} \mathcal{O}_{\mathbb{C P}^{4}}(5)\right)\right) .
$$

Since $\operatorname{ker}\left(d_{f}^{\prime \prime}\right)=H^{0}\left(D, f^{*} \mathcal{O}_{\mathbb{C P}^{4}}(5)\right)$, and $d_{f}^{\prime \prime}$ is surjective for all $f$, we have a short exact sequence

$$
0 \rightarrow \check{F}_{d}^{p} \rightarrow Y^{p} \stackrel{d^{\prime \prime}}{\rightarrow} Z^{p} \rightarrow 0
$$

Let

$$
R: Z^{p} \rightarrow Y^{p}
$$

denote the unique right inverse of $d^{\prime \prime}$ such that the image of $R_{f}$ is the $L^{2}$ complement of $\operatorname{ker}\left(d_{f}^{\prime \prime}\right)$. Let

$$
P: Y^{p} \rightarrow \check{F}_{d}^{p}
$$

denote the $L^{2}$ projection. Define a section $\hat{\xi}_{p}$ of $Y^{p}$ by

$$
\hat{\xi}_{p}(f)=f^{-1} \xi \text {. }
$$

Throughout the following, we use $\|\cdot\|$ to denote a context dependent norm. That is, for sections of Banach space bundles, $\|\cdot\|$ is the appropriate Banach space norm and, for operators, $\|\cdot\|$ is the appropriate operator norm.

Reformulating the definition of $\check{\xi}_{p}$ given in (22), we have

$$
\check{\xi}_{p}=P \hat{\xi}_{p}=\hat{\xi}_{p}-R_{f} \circ d_{f}^{\prime \prime}\left(\hat{\xi}_{p}\right) .
$$

We will argue that for $f$ close enough to $p$, we have

$$
\left\|R_{f} \circ d_{f}^{\prime \prime}\left(f^{-1} \xi\right)\right\|_{1, r} \leq \epsilon \operatorname{dist}\left(f, V_{p}^{Q}\right)
$$


for arbitrary epsilon. Here, $\operatorname{dist}(\cdot, \cdot)$ denotes an arbitrary distance function. Since $\xi$ vanishes transversely at $Q$, we know that $\hat{\xi}_{p}$ vanishes transversely on $V_{p}^{Q}$. That is, we know that

$$
\left\|f^{-1} \xi\right\|_{1, r} \geq \epsilon_{0} \operatorname{dist}\left(f, V_{p}^{Q}\right) .
$$

Choosing $\epsilon<\epsilon_{0}$, estimate (25) shows that after we perturb $\hat{\xi}_{p}$ by $R_{f} \circ d_{f}^{\prime \prime}\left(\hat{\xi}_{p}\right)$ to obtain $\check{\xi}_{p}$, it is still transverse and vanishes only on $V_{p}^{Q}$.

In order to prove estimate (25), we calculate $d_{f}^{\prime \prime}\left(f^{-1} \xi\right)$. Let $j$ denote the complex structure on $D$, let $J$ denote the complex structure on $\mathbb{C P}^{4}$ and let $I$ denote the complex structure on $\mathcal{O}_{\mathbb{C P}^{4}}(5)$. Assume that

$$
\bar{\partial} f=\eta_{f} \in\left(\check{E}_{p}\right)_{f} .
$$

We calculate

$$
\begin{aligned}
d_{f}^{\prime \prime}\left(f^{-1} \xi\right) & =\nabla^{f}\left(f^{-1} \xi\right)+I \nabla^{f}\left(f^{-1} \xi\right) \circ j \\
& =\nabla \xi \circ d u+I(\nabla \xi \circ d u) \circ j \\
& =\nabla \xi \circ d u+I\left(\nabla \xi \circ\left(-J \circ d u \circ j+\eta_{f}\right)\right) \circ j \\
& =I \nabla \xi \circ \eta_{f} \circ j .
\end{aligned}
$$

The third equality uses equation (26) and the fourth equality uses the holomorphicity of $\xi$ to cancel $I$ and $-J$.

Composition with $\nabla \xi$ defines a linear map

$$
\nabla \xi \circ: E_{p} \rightarrow Z^{p}
$$

Observe that for $f \in V_{p}^{Q}$ we have

$$
\left(E_{p}\right)_{f}=\left(E_{p}^{Q}\right)_{f} \subset \mathcal{E}_{f}^{Q}=L^{p}\left(D, \Omega^{0,1}\left(f^{*} T Q\right)\right) .
$$

Since $\xi$ vanishes on $Q$, we see that $\nabla \xi \circ$ maps $\left(E_{p}\right)_{f}$ to zero for $f \in V_{p}^{Q}$. By continuity, we have

$$
\left\|(\nabla \xi \circ)_{f}\right\| \leq C \operatorname{dist}\left(f, V_{p}^{Q}\right) .
$$

So, we infer from calculation (27) that

$$
\left\|d_{f}^{\prime \prime}\left(f^{-1} \xi\right)\right\| \leq C \operatorname{dist}\left(f, V_{p}^{Q}\right)\left\|\eta_{f}\right\| .
$$

Furthermore, we can assume a uniform bound

$$
\left\|R_{f}\right\| \leq C^{\prime}
$$

for all $f \in V_{p}$. For any $\epsilon^{\prime}>0$, we can choose $V_{p}$ so small that for all $f \in V_{p}$ we have

$$
\left\|\eta_{f}\right\| \leq \epsilon^{\prime}
$$

So, choosing $\epsilon^{\prime}$ such that $\epsilon^{\prime} C C^{\prime}<\epsilon$ and combining estimates (28), (29) and (30), we conclude estimate (25). This completes the proof of Lemma 17.

Proof of Lemma 18. The following proof is a generalization of the argument given in [19, Section 8, Proposition 8.8]. In the following, we will abbreviate

$$
T B:=T B_{D}\left(\mathbb{C} P^{4} / \mathbb{C} P_{\mathbb{R}}^{4}, d\right), \quad T B^{Q}:=T B_{D}\left(Q / Q_{\mathbb{R}}, d\right) .
$$

We continue to use the bundles $Y$ and $Z$ introduced in the proof of Lemma 17. Since $V_{p}^{Q}=\check{\xi}^{-1}(0) \subset V_{p}$, and by Lemma 17, $\check{\xi}$ is transverse to 0 , we conclude that $d \check{\xi}$ induces an isomorphism

$$
\operatorname{det}\left(T V_{p}^{Q}\right) \stackrel{\sim}{\rightarrow} \operatorname{det}\left(T V_{p}\right) \otimes \operatorname{det}\left(\check{F}_{d}\right)^{*}
$$




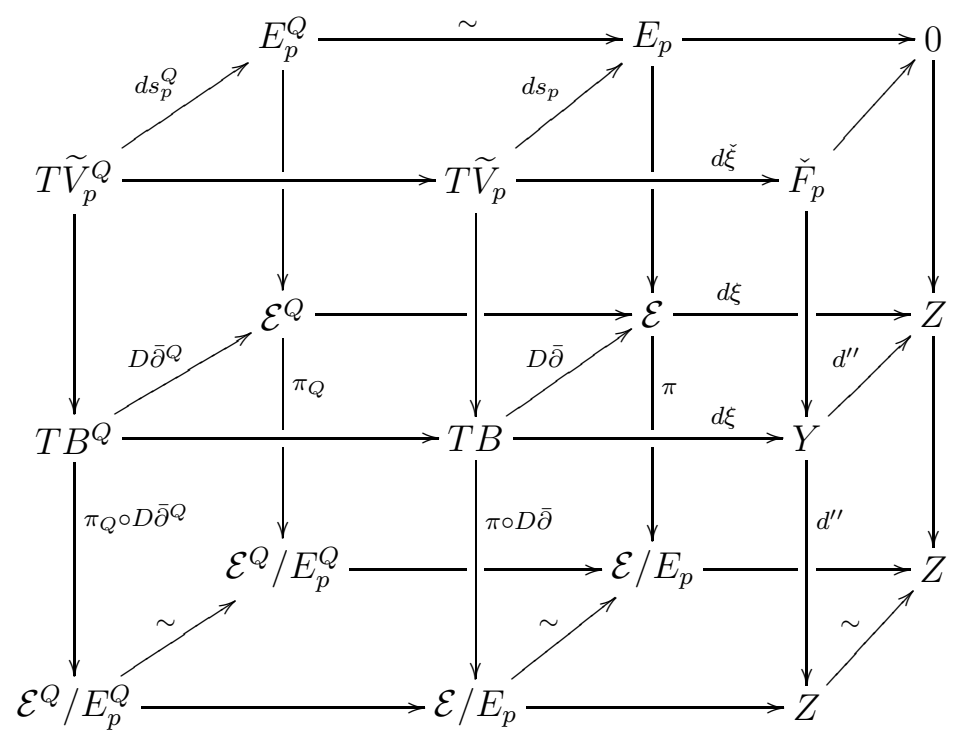

FiguRE 3

along $V_{p}^{Q}$. Using the fact that by construction $E_{p}^{Q}=\left.E_{p}\right|_{V_{p}^{Q}}$, we can tensor the above isomorphism with $\operatorname{det}\left(E_{p}^{Q}\right)^{*}=\operatorname{det}\left(E_{p}\right)^{*}$ to obtain an isomorphism

$$
\mathcal{L}_{\mathcal{K}^{Q}} \stackrel{d \xi}{\longrightarrow} \mathcal{L}_{\mathcal{K}} \otimes \operatorname{det}\left(\check{F}_{d}\right)^{*} .
$$

The lemma will follow if we show that isomorphism (31) respects the canonical orientations of each of the three deteminant bundles $\mathcal{L}_{\mathcal{K}^{Q}}, \mathcal{L}_{\mathcal{K}}$, and $\operatorname{det}\left(\check{F}_{d}\right)$. For this purpose, we introduce the commutative diagram of vector bundles over $\tilde{V}_{p}^{Q}$ of Figure 3. Here, we implicitly consider the restrictions of $T B, T B^{Q}, \mathcal{E}, \mathcal{E}^{Q}, T V_{p}$ and $E_{p}$ to $V_{p}^{Q}$. We denote by $\pi$ and $\pi_{Q}$ the canonical projections to the quotient.

In order for all squares in the diagram of Figure 3 to commute, we must choose the complex connection $\nabla$ on $\mathcal{O}_{\mathbb{C} P^{4}}(5)$ carefully. Indeed, $\left.d \xi\right|_{Q}$ induces an isomorphism of complex vector bundles

$$
\left.d \xi\right|_{Q}:\left.T \mathbb{C} P^{4}\right|_{Q} / T Q \stackrel{\sim}{\longrightarrow} \mathcal{O}_{\mathbb{C} P^{4}}(5) .
$$

So, we are free to choose $\nabla$ to be the complex connection induced by $\left.d \xi\right|_{Q}$ from the connection on $\left.T \mathbb{C} P^{4}\right|_{Q} / T Q$ induced by the Levi-Civita connection of $\mathbb{C} P^{4}$ with respect to the standard Kähler metric. This ensures that

$$
d^{\prime \prime} \circ d \xi=d \xi \circ D \bar{\partial}
$$

i.e., that the middle right horizontal square of the diagram commutes. Moreover, the proof of Lemma 17 implies that

$$
i_{F} \circ d \check{\xi}=d \xi \circ i
$$

i.e., that the front upper right vertical square of the diagram commutes. The commutativity of the remaining squares is straightforward.

Moreover, all columns and rows of Figure 3 are exact. We think of the diagram as an exact square of two-step complexes. Applying the determinant functor to the long exact sequence of a short exact sequence of two step complexes yields an 


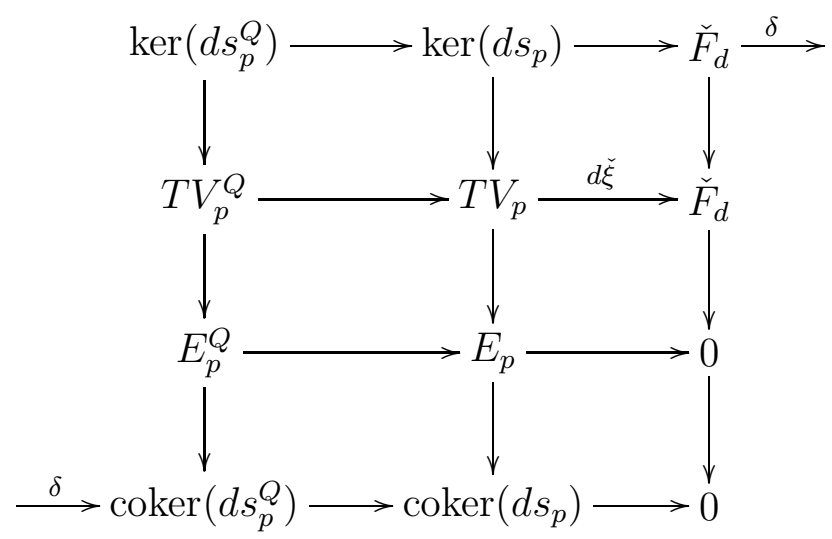

FIGURE 4

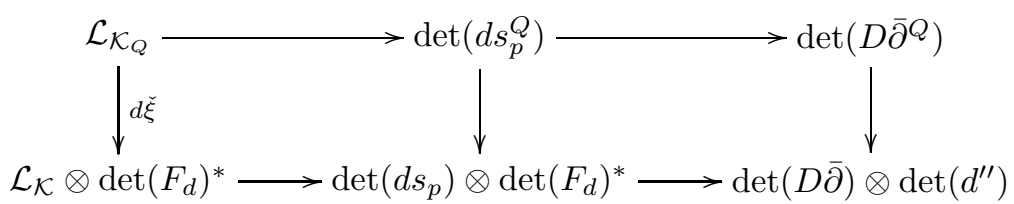

FiguRE 5

isomorphism of determinant bundles. In particular, the five nontrivial rows and columns of Figure 3 give isomorphisms

$$
\begin{aligned}
\operatorname{det}\left(d s_{p}^{Q}\right) & \simeq \operatorname{det}\left(D \bar{\partial}^{Q}\right), \quad \operatorname{det}\left(d s_{p}\right) \simeq \operatorname{det}(D \bar{\partial}), \quad \operatorname{det}\left(\check{F}_{d}\right) \simeq \operatorname{det}\left(d^{\prime \prime}\right), \\
\operatorname{det}(D \bar{\partial}) & \simeq \operatorname{det}\left(D \bar{\partial}^{Q}\right) \otimes \operatorname{det}\left(d^{\prime \prime}\right)^{*}, \quad \operatorname{det}\left(d s_{p}^{Q}\right) \simeq \operatorname{det}\left(d s_{p}\right) \otimes \operatorname{det}\left(\check{F}_{d}\right)^{*} .
\end{aligned}
$$

Taking determinants of the rows and columns of the diagram of Figure 4, we obtain isomorphisms

$$
\begin{gathered}
\operatorname{det}\left(d s_{p}^{Q}\right) \simeq \mathcal{L}_{\mathcal{K}_{Q}}, \quad \operatorname{det}\left(d s_{p}\right) \simeq \mathcal{L}_{\mathcal{K}}, \\
\mathcal{L}_{\mathcal{K}_{Q}} \simeq \mathcal{L}_{\mathcal{K}} \otimes \operatorname{det}\left(\check{F}_{d}\right)^{*}, \quad \operatorname{det}\left(d s_{p}^{Q}\right) \simeq \operatorname{det}\left(d s_{p}\right) \otimes \operatorname{det}\left(\check{F}_{d}\right)^{*} .
\end{gathered}
$$

Clearly the last isomorphism of (32) agrees with the last isomorphism of (33). Putting all the isomorphisms of (32) and (33) together, we obtain the diagram of Figure 5. The left square of Figure 5 commutes by the commutativity of Figure 4 . The right square of Figure 5 commutes by the commutativity of Figure 3 The rows of Figure 5 preserve orientation by definition. The right column of Figure 5 preserves orientation by [19, Section 8, Proposition 8.4] and the compatibility of $\mathfrak{s}, \mathfrak{p}$, and $\mathfrak{p}^{\prime}$. By commutativity the left column of Figure 5 also preserves orientation, completing the proof.

\section{Multiple COVer formula}

6.1. Local $\mathbb{C P}^{1}$. Consider $\mathbb{C P}^{1}$ with the anti-holomorphic involution $c$ defined in Section 1.3.3. The involution $c$ lifts canonically to $\mathcal{O}_{\mathbb{C P}^{1}}(-1)$. The total space of the rank 2 bundle

$$
\mathcal{O}_{\mathbb{C P}^{1}}(-1) \oplus \mathcal{O}_{\mathbb{C P}^{1}}(-1) \rightarrow \mathbb{C P}^{1}
$$


with the associated anti-holomorphic involution may be viewed as a local model for a rational curve in $Q$.

The local disk invariants of $\mathbb{C P}^{1}$ are, by definition,

$$
L_{d}^{\text {disk }}=\int_{\widetilde{M}_{D}\left(\mathbb{C P}^{1} / \mathbb{C P}_{\mathbb{R}}^{1}, d\right)} e\left(G_{d} \oplus G_{d}\right),
$$

where $G_{d}$ is the real vector bundle over $\widetilde{M}_{D}\left(\mathbb{C P}^{1} / \mathbb{C P}_{\mathbb{R}}^{1}, d\right)$ with fiber

$$
\left.G_{d}\right|_{\left[f:(D, \partial D) \rightarrow\left(\mathbb{C P}^{1}, \mathbb{C P}_{\mathbb{R}}^{1}\right)\right]}=H^{1}\left(C, \tilde{f}^{*} \mathcal{O}_{\mathbb{C P}^{1}}(-1)\right)_{\mathbb{R}}
$$

Here,

$$
\left[\tilde{f}: C \rightarrow \mathbb{C P}^{1}\right] \in \bar{M}_{\mathbb{R}}\left(\mathbb{C P}^{1}, d\right)
$$

is the stable rational map obtained from the stable disk map via reflection. As before, we consider only the $d$ odd case.

Proposition 19. For $d$ odd, $L_{d}^{\text {disk }}=2 d^{-2}$.

The factor of 2 on the right occurs since the original $\mathbb{C P}^{1}$ consists of 2 disks. Hence, Proposition 19 may be viewed as the calculation of twice the multiple cover contribution of a single disk.

We emphasize that $d$ is the degree of the stable rational map obtained from the stable disk map by reflection. In particular, the degree of a map $f$ in $\widetilde{M}_{D}\left(\mathbb{C P}^{1} / \mathbb{C P}_{\mathbb{R}}^{1}, d\right)$ restricted to $\partial D$ may be any odd integer less than or equal to $d$. This is necessary in order to perform the analogue of the construction of Proposition 11 for target space $\mathbb{C} P^{1}$, as the diffeomorphisms $c_{I}$ do not preserve boundary degree. In this regard, our approach differs from the approach of [10.

6.2. Torus action. Let the torus $\mathbf{T}$ act on $\mathbb{C P}^{1}$ by

$$
\xi \cdot[u, v]=[\xi u, \bar{\xi} v] .
$$

The fixed points are

$$
\zeta_{1}=[1,0], \quad \zeta_{2}=[0,1]
$$

with tangent weights $2 \lambda$ and $-2 \lambda$, respectively. The $\mathbf{T}$-action preserves $\mathbb{C P}_{\mathbb{R}}^{1}$, and therefore it determines a translation action on the moduli space of disks $\widetilde{M}_{D}\left(\mathbb{C P}^{1} / \mathbb{C P}_{\mathbb{R}}^{1}, d\right)$.

The $\mathbf{T}$-action lifts to $\mathcal{O}_{\mathbb{C P}^{1}}(-1)$ with fiber weights $-\lambda$ and $\lambda$ over $\zeta_{1}$ and $\zeta_{2}$, respectively, the unique lift which respects the real structure on $\mathcal{O}_{\mathbb{C P}^{1}}(-1)$.

\subsection{Proof of Proposition 19. We give two different proofs.}

First proof. The invariants $L_{d}^{\text {disk }}$ are calculated by $\mathbf{T}$-equivariant localization. Since the steps are so similar to the proof of Theorem 1, we give an abbreviated account.

As before, to each map $[f] \in \bar{M}_{D}\left(\mathbb{C P}^{1} / \mathbb{C P}_{\mathbb{R}}^{1}, d\right)^{\mathbf{T}}$ we associate an intersection disk. The intersection disk terms $I\left(\zeta_{1}, p\right)$ and $I\left(\zeta_{2}, p\right)$ are both equal to

$$
(-1)^{\frac{p-1}{2}} \frac{2^{1-e}}{p} \frac{(p ! !)^{2}}{p !}
$$


The sign $(-1)^{\frac{p-1}{2}}$ comes from the normal bundle to the disk map, just as in the proof of Lemma 6. We do not have to calculate the orientation of $H^{1}\left(C, \tilde{f}^{*} \mathcal{O}_{\mathbb{C P}^{1}}(-1)\right)_{\mathbb{R}}$ because it appears twice.

Givental's equivariant correlator $S_{L}$ for the local geometry is defined by

$$
S_{L}(T, \hbar)=\sum_{r \geq 0} e^{(H / \hbar+r) T} e_{2 *}\left(\frac{c_{\text {top }}\left(H^{1}(\mathcal{O}(-1)) \oplus H^{1}(\mathcal{O}(-1))\right)}{\hbar-\psi_{2}}\right) \in H_{\mathbf{T}}^{*}\left(\mathbb{C P}^{1}\right),
$$

where

$$
e_{2}: \bar{M}_{0,2}\left(\mathbb{C P}^{1}, d\right) \rightarrow \mathbb{C P}^{1}
$$

is the evaluation map. The calculation of $S_{L}$ in [6] is much easier than $S_{Q}$,

$$
S_{L}(T, \hbar)=\sum_{r \geq 0} e^{r T} \frac{\prod_{s=0}^{r-1}(H+s \hbar)^{2}}{\prod_{s=1}^{r}(H-\lambda+s \hbar)(H+\lambda+s \hbar)} .
$$

No mirror transform is needed.

The local disk potential $\mathcal{F}_{L}^{\text {disk }}$ is defined by summing over odd degrees,

$$
\mathcal{F}_{L}^{\text {disk }}=\sum_{d \text { odd }} e^{d T / 2} L_{d}^{d i s k}
$$

As before,

$$
\mathcal{F}_{L}^{\text {disk }}=\sum_{p \text { odd }}\left\langle S_{L}\left(T, \frac{2}{p} \lambda\right),\left[\zeta_{1}\right]\right\rangle \cdot I\left(\zeta_{1}, p\right)+\sum_{p \text { odd }}\left\langle S_{L}\left(T,-\frac{2}{p} \lambda\right),\left[\zeta_{2}\right]\right\rangle \cdot I\left(\zeta_{2}, p\right) .
$$

Evaluation yields

$$
\mathcal{F}_{L}^{\text {disk }}=2 \sum_{r \geq 0} \sum_{p \text { odd }} e^{\left(\frac{p}{2}+r\right) T} \frac{2^{1-p-2 r}}{(p+2 r)^{2}} \frac{(-1)^{\frac{p-1}{2}}}{p} \frac{(p ! !)^{2}}{p ! r !} \frac{\prod_{i=1}^{r}(p+2 i)^{2}}{\prod_{i=1}^{r}(p+i)} .
$$

The proof of the proposition is concluded by extracting the $e^{d T / 2}$ terms on the right and executing the sum,

$$
\begin{aligned}
L_{d}^{d i s k} & =\frac{2}{d^{2}} \sum_{1 \leq p \text { odd } \leq d} \frac{(d ! !)^{2}}{2^{d-1}} \frac{(-1)^{\frac{p-1}{2}}}{\left(\frac{d-p}{2}\right) !\left(\frac{d-p}{2}+p\right) ! p} \\
& =\frac{2}{d^{2}} .
\end{aligned}
$$

Remarkably, the binomial identity required is exactly (12)!

Second proof. Although each copy of $\mathcal{O}_{\mathbb{C P}^{1}}(-1)$ admits a unique real $\mathbf{T}$-action, there is some freedom in the choice of action on the sum

$$
\mathcal{O}_{\mathbb{C P}^{1}}(-1) \oplus \mathcal{O}_{\mathbb{C P}^{1}}(-1)
$$


Indeed, we can choose the action induced by the isomorphism of vector bundles with real structure,

$$
\mathcal{O}_{\mathbb{C P}^{1}}(-1) \oplus \mathcal{O}_{\mathbb{C P}^{1}}(-1) \simeq \mathcal{O}_{\mathbb{C P}^{1}}(-1) \otimes_{\mathbb{C}} W_{\lambda}
$$

Here, $W_{\lambda}=V_{\lambda} \otimes \mathbb{C}$, and $V_{\lambda}$ is the real representation of the torus of weight $\lambda$.

With the $\mathbf{T}$-action determined by the right side of (34), the localization contribution from any reducible open stable map vanishes. Indeed, we work this out explicitly for a torus fixed open stable map $f$ with reducible domain $D$ consisting of one disk component $D_{o}$ and one sphere component $D_{c}$. The general case is similar.

Denote the single node of $D$ by $z$. Consider the exact sequence

$$
\begin{aligned}
0 & \rightarrow H^{0}\left(z, f^{*} \mathcal{O}_{\mathbb{C P}^{1}}(-1) \otimes W_{\lambda}\right) \rightarrow H^{1}\left(D, f^{*} \mathcal{O}_{\mathbb{C P}^{1}}(-1) \otimes W_{\lambda}\right) \\
& \rightarrow H^{1}\left(D_{o}, f^{*} \mathcal{O}_{\mathbb{C P}^{1}}(-1) \otimes W_{\lambda}\right) \oplus H^{1}\left(D_{c}, f^{*} \mathcal{O}_{\mathbb{C P}^{1}}(-1) \otimes W_{\lambda}\right) \rightarrow 0 .
\end{aligned}
$$

We claim that $H^{0}\left(z, f^{*} \mathcal{O}_{\mathbb{C P}^{1}}(-1) \otimes W_{\lambda}\right)$ contains a zero weight space, and therefore so does $H^{1}\left(D, f^{*} \mathcal{O}_{\mathbb{C P}^{1}}(-1) \otimes W_{\lambda}\right)$, which immediately implies that the localization contribution of $[f]$ vanishes. Indeed,

$$
H^{0}\left(z, f^{*} \mathcal{O}_{\mathbb{C} P^{1}}(-1) \otimes W_{\lambda}\right)
$$

is just the fiber of $f^{*} \mathcal{O}_{\mathbb{C} P^{1}}(-1) \otimes W_{\lambda}$ at $z$. Denoting by $\mathbb{C}_{\lambda}$ the complex representation of the torus of weight $\lambda$ we have

$$
W_{\lambda} \simeq \mathbb{C}_{\lambda} \oplus \mathbb{C}_{-\lambda}, \quad f^{*} \mathcal{O}_{\mathbb{C} P^{1}}(-1)_{z} \simeq \mathbb{C}_{ \pm \lambda}
$$

Hence,

$$
H^{0}\left(z, f^{*} \mathcal{O}_{\mathbb{C} P^{1}}(-1) \otimes W_{\lambda}\right)=\mathbb{C}_{0} \oplus \mathbb{C}_{ \pm 2 \lambda},
$$

as claimed.

It remains to calculate the localization contribution from the single torus fixed irreducible open stable map of degree $d$. This is easily seen to be $\frac{2}{d^{2}}$.

The two proofs of Proposition 19 together provide a geometric evaluation of the binomial sum of Lemma 8 .

6.4. Integrality. The virtual disk counts $n_{d}^{\text {disk }}$ of Definition 2 have not yet been proven to be integers. However, Table 2 in Section 7 provides substantial evidence for the integrality claim.

\section{TABLES}

Table 1 shows the value of the disk Gromov-Witten invariant $N_{d}^{\text {disk }}$ for small $d$. Table 2 shows the corresponding virtually enumerative invariants $n_{d}^{\text {disk }}$. Recall the virtual counts $n_{d}^{\text {real }}$ of real curves in $Q$ differ by a factor of $1 / 2$,

$$
n_{d}^{r e a l}=\frac{1}{2} n_{d}^{d i s k} .
$$


TABLE 1

\begin{tabular}{l|l}
$d$ & $N_{d}^{\text {disk }}$ \\
\hline 1 & 30 \\
3 & $\frac{4600}{3}$ \\
5 & $\frac{5441256}{5}$ \\
7 & $\frac{47823842250}{49}$ \\
9 & $\frac{28973369597500}{27}$ \\
11 & $\frac{160812279574853640}{121}$ \\
13 & $\frac{301152359429255569200}{169}$ \\
15 & 2528247216911976710478 \\
17 & $\frac{1081454384062665012504422250}{289}$ \\
19 & $\frac{2066166201384849550431238897500}{361}$ \\
21 & $\frac{440336544802747748968402664543390}{49}$ \\
23 & $\frac{7625558614788648016004683159051585650}{529}$ \\
25 & $\frac{2942308498496733293257158606365620128756}{125}$ \\
27 & $\frac{9481608375404186315963625791852891724001750}{243}$ \\
29 & $\frac{55101515400393595065761084565358564820821590000}{841}$ \\
&
\end{tabular}

TABLE 2

\begin{tabular}{c|l}
$d$ & $n_{d}^{\text {disk }}$ \\
\hline 1 & 30 \\
3 & 1530 \\
5 & 1088250 \\
7 & 975996780 \\
9 & 1073087762700 \\
11 & 1329027103924410 \\
13 & 1781966623841748930 \\
15 & 2528247216911976589500 \\
17 & 3742056692258356444651980 \\
19 & 5723452081398475208950800270 \\
21 & 8986460098015260183028517362890 \\
23 & 14415044640432226873354788580437780 \\
25 & 23538467987973866346057268850924917500 \\
27 & 39018964507836157678862657579522297754750 \\
29 & 65519043282275380577599387116954298241167170
\end{tabular}

Acknowledgements. We thank P. Biran, T. Graber, D. Kazhdan, N. Nekrasov, L. Polterovich, P. Seidel, E. Shustin, G. Tian, R. Vakil, E. Zaslow, and A. Zinger for related conversations. 
The second author would like to thank E. Farjoun and the Hebrew University of Jerusalem for their warm hospitality during the preparation of the paper. The first author was partially supported by a Packard Foundation fellowship and NSF grant DMS-0500187. The second author was partially supported by NSF grant DMS0111298. The third author was partially supported by NSF grant PHY-0503584.

\section{REFERENCES}

[1] P. Aspinwall and D. Morrison, Topological field theory and rational curves, Comm. Math. Phys. 151 (1993), 245-262. MR1204770 (94h:32033)

[2] P. Candelas, X. de la Ossa, P. Green and L. Parkes, A pair of Calabi-Yau manifolds as an exactly soluble superconformal field theory, Nuclear Physics B359 (1991), 21-74.

[3] K. Fukaya, Y.-G. Oh, H. Ohto and K. Ono, Lagrangian intersection Floer theory, anomaly and obstruction, Kyoto University, preprint, 2006.

[4] K. Fukaya and K. Ono, Arnold conjecture and Gromov-Witten invariant, Topology 38 (1999), no. 5, 933-1048. MR 1688434 (2000j:53116)

[5] A. Givental, Equivariant Gromov-Witten invariants, Int. Math. Res. Notices 13 (1996), 613663. MR.1408320 (97e:14015)

[6] A. Givental, Elliptic Gromov-Witten invariants and the generalized mirror conjecture, math.AG/9803053.

[7] T. Graber and E. Zaslow, Open string Gromov-Witten theory: calculation and a mirror theorem, hep-th/0109075.

[8] H. Hofer, K. Wysocki and E. Zehnder, A General Fredholm Theory I: A Splicing-Based Differential Geometry, J. Eur. Math. Soc. 9 (2007), 841-876. MR2341834

[9] H. Hofer, K. Wysocki and E. Zehnder, A General Fredholm Theory II: Implicit Function Theorems, arXiv:0705.1310.

[10] S. Katz and M. Liu, Enumerative geometry of stable maps with Lagrangian boundary conditions and multiple covers of the disk, Adv. Theor. Math. Phys. 5 (2002), 1-49. MR.1894336 (2003e:14047)

[11] M. Kontsevich, Enumeration of rational curves via torus actions, in The moduli space of curves (R. Dijkgraaf, C. Faber, and G. van der Geer, eds.), Birkhäuser, 1995, 335-368. MR.1363062 (97d:14077)

[12] M. Kontsevich, Homological algebra of mirror symmetry. Proceedings of the International Congress of Mathematicians, Vol. 1, 2 (Zurich, 1994), 120-139, Birkhäuser, Basel, 1995. MR:1403918 (97f:32040)

[13] B. Lian, K. Liu, and S.-T. Yau, Mirror principle I, Asian J. Math. 4 (1997), 729-763. MR1621573 (99e:14062)

[14] M. Liu, Moduli of J-holomorphic curves with Lagrangian boundary conditions and open Gromov-Witten invariants for a $S^{1}$-equivariant pair, math.SG/0210257.

[15] H. Ooguri and C. Vafa, Knot invariants and topological strings, Nucl. Phys. B 577 (2000), 419-438. MR.1765411(2001i:81254)

[16] R. Pandharipande, Rational curves on hypersurfaces [after A. Givental], Séminaire Bourbaki, 50ème année, 1997-1998, Astérique, no. 848. MR.1685628 (2000e:14094)

[17] Y. Ruan and G. Tian, A mathematical theory of quantum cohomology, J. Differential Geom. 42 (1995), no. 2, 259-367. MR.1366548 (96m:58033)

[18] P. Seidel, personal communication based on a remark of D. Joyce and a talk of K. Fukaya at Northwestern in spring 2004.

[19] J. Solomon, Intersection theory on the moduli space of holomorphic curves with Lagrangian boundary conditions, math.SG/0606429.

[20] J. Solomon, Virtual manifolds, to appear.

[21] J. Walcher, Opening mirror symmetry on the quintic, hep-th/0605162.

[22] J.-Y. Welschinger, Invariants of real symplectic 4-manifolds and lower bounds in real enumerative geometry, Invent. Math. 162 (2005), 195-234. MR.2198329 (2006i:53123)

[23] J.-Y. Welschinger, Spinor states of real rational curves in real algebraic convex 3-manifolds and enumerative invariants, Duke Math. J. 127 (2005), 89-121. MR2126497 (2005k:14121) 
[24] K. Wehrheim and C. Woodward, Orientations for pseudo-holomorphic quilts, preprint.

[25] E. Witten, Chern-Simons gauge theory as a string theory, The Floer memorial volume, 637-678, Progr. Math., 133, Birkhäuser, Basel, 1995. arXiv: hep-th/9207094. MR1362846 $(97 \mathrm{j}: 57052)$

Department of Mathematics, Princeton University, Princeton, New Jersey 08544

E-mail address: rahulp@math.princeton.edu

School of Mathematics, Institute for Advanced Study, Princeton, New Jersey 08540

E-mail address: jake@ias.edu

Current address: Department of Mathematics, Princeton University, Princeton, New Jersey 08544

E-mail address: jake@math.princeton.edu

School of Natural Science, Institute for Advanced Study, Princeton, New Jersey 08540

E-mail address: walcher@ias.edu 